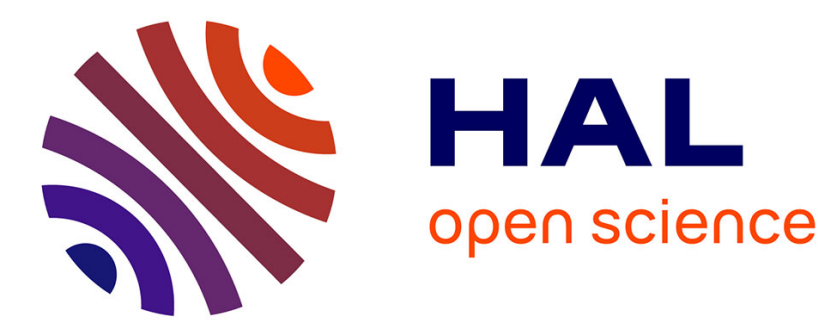

\title{
In-Salah 1904/Tamanrasset 1905 : les deux soumissions des Touaregs Kel-Ahaggar
}

Paul Pandolfi

\section{To cite this version:}

Paul Pandolfi. In-Salah 1904/Tamanrasset 1905: les deux soumissions des Touaregs Kel-Ahaggar.

Cahiers d'études africaines, 1998, 38, pp.41 - 83. 10.3406/cea.1998.1976 . hal-03174253

\section{HAL Id: hal-03174253 \\ https://hal.science/hal-03174253}

Submitted on 19 Mar 2021

HAL is a multi-disciplinary open access archive for the deposit and dissemination of scientific research documents, whether they are published or not. The documents may come from teaching and research institutions in France or abroad, or from public or private research centers.
L'archive ouverte pluridisciplinaire HAL, est destinée au dépôt et à la diffusion de documents scientifiques de niveau recherche, publiés ou non, émanant des établissements d'enseignement et de recherche français ou étrangers, des laboratoires publics ou privés. 


\title{
In-Salah 1904/Tamanrasset 1905 : les deux soumissions des Touaregs Kel-Ahaggar \\ In: Cahiers d'études africaines. Vol. 38 N¹49. 1998. pp. 41-83.
}

\section{Résumé \\ Résumé}

Dans les premières années du XXe siècle, suite à la défaite militaire connue par les Touaregs Kel-Ahaggar au combat de Tit (1902), la France s'installe au Sahara central. Dès lors un des principaux obstacles à la liaison Maghreb/Soudan est levé. Longtemps l'histoire consacrée à cette période cruciale s'est cantonnée dans un discours hagiographique et répétitif. L'examen de documents d'archives jusqu'ici peu exploités permet de mieux cerner les stratégies mises en œuvre et les lignes politiques divergentes qui se sont alors manifestées — au moins au niveau tactique — tant chez les Kel-Ahaggar que chez les militaires français. La reconstruction de ce pan d'histoire met au jour non seulement les alliances et les divisions propres aux deux camps en présence mais également l'empirisme qui a souvent dominé la politique de pénétration coloniale dans cette région.

\begin{abstract}
Abstract

In-Salah, 1904/Tamanrasset, 1905: Two acts of submission by the Kel-Ahaggar Tuareg. - Early in the 20th century, following the battle of Tit in 1902, when the French defeated the Kel-Ahaggar Tuareg, France extended its authority into the central Sahara. This did away with one of the major obstacles to contacts between North Africa and the Sudan. For a longtime, the history of this key period has been locked up inside a repetitious, hagiographie discourse. Archives, which have not been much studied to date, shed light on the strategies and divergent political lines pursued, at least tactically, by the Kel-Ahaggar and French military officers. Reconstructing this history brings to light the alliances and divisions on each side; and it draws attention to the empiricism that often prevailed in colonial policy about how to penetrate this region.
\end{abstract}

Citer ce document / Cite this document :

Pandolfi Paul. In-Salah 1904/Tamanrasset 1905 : les deux soumissions des Touaregs Kel-Ahaggar. In: Cahiers d'études africaines. Vol. 38 N¹49. 1998. pp. 41-83.

doi : 10.3406/cea.1998.1976

http://www.persee.fr/web/revues/home/prescript/article/cea_0008-0055_1998_num_38_149_1976 


\title{
Paul Pandolfi
}

\section{In-Salah 1904/Tamanrasset 1905 :}

\author{
les deux soumissions \\ des Touaregs Kel-Ahaggar
}

Pour l'essentiel l'histoire de la conquête du Sahara central s'est jusqu'à présent cantonnée dans une répétition hagiographique. Si cette littérature a connu son heure de gloire dans les années 1920-1950, elle est cependant loin d’avoir totalement disparue comme le démontrent encore quelques publications récentes'. Mouvement inverse et salutaire, sous l'impulsion d'ethnologues plus que d'historiens, depuis quelques décennies, s'est aussi opéré un renversement de perspective ${ }^{2}$. L'histoire de l'expansion coloniale au Sahara tente désormais de prendre en compte le point de vue des populations autochtones et d'intégrer autant que faire se peut la situation politico-économique connue par ces populations. Mais, même dans cette perspective, reste encore plus ou moins sous-entendu que la politique coloniale française se présentait à cette époque (fin $X I X^{\circ}$-début $x X^{*}$ siècle) comme un bloc uniforme. Or, rien n'est moins sûr. Si l'on abandonne les stéréotypes "gloricux» de la littérature coloniale, si l'on se réfère aux archives contemporaines de l'avancée française au Sahara ${ }^{3}$, il apparaît alors que de profondes divergences séparaient les principaux acteurs côté français - de cet épisode historique. Certes, un accord de fond les réunissait: le Sahara devait devenir une terre française permettant ainsi l'unification en un même ensemble des possessions de la France en Afrique du Nord et en Afrique occidentale. Mais cet accord stratégique n'annule en rien les oppositions tactiques quant à la manière même d'atteindre un tel objectif. Ces oppositions propres au « camp français " se sont notamment manifestées entre des hommes ayant joué un rôle clé lors de cette

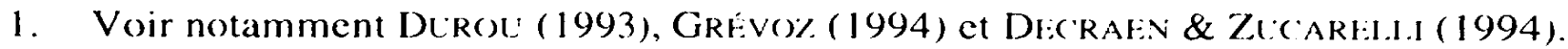

2. Dans le cas des Kel-Ahaggar, nous pensons plus particulièrement aux travaux de GAST (1986 et 1993 notamment) et Botrgitor (1979 et 1984, repris in 1995 : 285-341).

3. Ces archives présentent un indéniable avantage. Elles ne s'en tiennent pas à la vision hagiographique qui va très vite dominer par la suite l'écriture de cet épisode historique. Elles ont pourtant été assez peu utilisées jusqu'ici. D'où notre parti pris, dans cette étude, de nombreuses et longues citations. 
phase historique qui au début de ce siècle déboucha sur la soumission des Kel-Ahaggar. Aussi l’histoire de cette période cruciale ne peut sécrire sans tenir compte de ce fait. Il ne sagit done pas de sombrer ici dans l’anecolotique, mais bien d’évaluer en quoi des contradictions secondaires mais présentes au sein même des deux camps ont influé sur le cours de cette période historique. Tâche d'autant plus nécessaire que nombre de points généralement admis comme des données incontestables méritent, au vu de certains documents d'archives. un réexamen critique. Ainsi, dans la littérature consacrée à cette séquence historique, deux dates dominent. En 1902. le combat de Tit marque la défaite militaire des Kel-Ahaggar et ouvre ainsi leur territoire à l'avancée française. Deux ans plus tard. à In-Salah, aurait lieu la soumission des Kel-Ahaggar. Le capitaine Métois y reçoit Mûsa ag̣g Amastân et le reconnatit comme amenâkal des KelAhaggar. Or, il nous paraît qu“il y a peut-être là une lecture trop hâtive de ceśsévénements majeurs. Mûsa agg Amastân, en fin politique sachant exploiter les divisions du camp adverse. a pu ainsi présenter sa venue à In-Salah comme une négociation où il avait préservé l’essentiel et non comme une soumission en bonne et due formet.

Lambiguité de la rencontre d'In-Salah (1904) et de l'accord qui y fut conclu lui a de fait permis d'entretenir cette illusion jusqu'en 1905. Laperrine l'a très vite compris et c'est bien pour cela que son opposition à Métois devient alors très virulente. D’où aussi l'absolue nécessité de la tournée entreprise par Dinaux en 1905. Si ce dernier tient à reconnaître à nouveau Mûsa comme amenákal, à préciser aux notables Kel-Ahaggar les conditions de leur soumission. c'est bien parce qu'In-Salah n'avait pas constitué, tant aux yeux de Laperrine qu'aux yeux de Mûsa. un véritable acte de soumission. Dès lors, la question qui a intrigué nombre d'observateurs (Mûsa fut-il reconnu à In-Salah en 1904 ou à Tamanrasset en 1905 ?) se pose en des termes différents.

Enfin, dernier point. mais non le moindre. il faut aussi réenvisager le rapport Mûsa/Atici. Un des thèmes récurrents de la littérature hagiogratphique, mais aussi de sérieux travaux scientifiques, est bien celui de la division des Kel-Ahaggar, d'une opposition irréductible entre deux camps, entre deux lignes politiques respectivement représentées par Mûsa et par Atici. Il y a là une contradiction interne qui nous semble incontestable. Mais elle n’a peut-être pas été aussi permanente. aussi systématique et virulente qu'on l’a généralement dit. Il n’est pas sûr, par exemple. que dès 1904 Mûsal. nommé amenúkal par les autorités coloniales, ait supplanté

4. Bourgeot avait déja releve ce point capital : "ll est fort probahle que Músa el ses partisams ont cru pendam un temps que ce tratite ratifiatit an aceord entre deux puissances pellitigues soucieuses d'instadurer un climat de patix dans la region. Músa el les siens. hommes libres. aristocrates de surcoroit. admedlatent lat suprématic militaire de la france salms pour antant se comsidérer comme ses

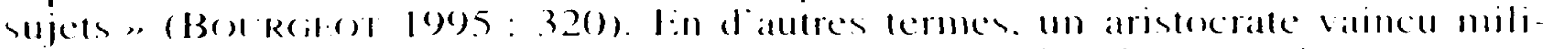
tatrement peut devenir le protégé mais non le tributaire de som vaingueur 
Atici auprès des Kel-Ahaggar. Il est significatif quà cette date encore Mûsa, tout en jouant sa propre carte (et en cela il est aussi de fait le concurrent d'Atici), prend également soin de se présenter comme un porteparole des Kel-Ahaggar dont le chef reste Atici lui-même.

En somme, le jeu complexe qui se déroule alors est loin de se réduire à un schéma simpliste. Il n’y a pas d'un côté un camp français uni sous la houlette d'un chef charismatique (Laperrine) et de l'autre une confédération touarègue incapable de présenter la moindre unité face aux menées coloniales. Nombre de contradictions secondaires tant dans le camp touareg que dans le camp français se révèlent, selon les circonstances et les enjeux, plus ou moins présentes, plus ou moins efficientes. C'est donc à une relecture de cette période cruciale que nous nous attachons ici.

\section{L'encerclement (1880-1900)}

«Partout où nous allons... nous nous heurtons à vous, chez. les Ouderna du Sud tunisien, aux portes de Ghadamès, c'est à vous que nous avons affaire: au Nefzaoua c'est à vous aussi, et dans le Sahara jusqu'à Figuig, nous vous trouvons partout : avec vous ces contrées-lá nous sont fermées, et nous sommes comme murés chè nous » (in Gast 1993: 50). Ainsi s’exprime un des membres de la délégation touarègue qui rencontre le général de La Roque en 1890 à Biskra. Ce constat d'encerclement, d'asphyxie programmée. se renforcera encore durant la dernière décennie du XIX" siècle. Au nord, après avoir occupé le M\%ab (1881), Ouargla, Touggourt et El Oued (1885), après avoir édifié une importante ligne de forts sahariens (Fort Lallemand, Fort Miribel, Fort Mac-Mahon...) et consolidé sa présence dans le Sud tunisien, la France s'empare d'In-Salah (1899) et, en 190(), contrôle le Tidikelt. Cette poussée septentrionale se combine avec une avancée le long du fleuve Niger (Bamako 1883, Tombouctou 1894, Say $1896 \ldots$ ) qui permet aux forces françaises de s’implanter aux marges méridionales du "pays " touareg. Tant au nord qu'au sud de la zone occupée par les Touaregs du Sahara central (Kel-Ahaggar et KelAjjer) ces événements traduisent une extension et une consolidation de l'avancée française. Il y a là, conséquence majeure du massacre de la mission Flatters, une nouvelle option stratégique ouvertement revendiquée par les autorités françaises : plutôt que de tenter des traversées militaires et/ou civiles, on préférera désormais imposer un véritable blocus économique aux Kel-Ahaggar en s'emparant des oasis du Touat-Tidikelt notamment. Juste après le massacre de la mission Flatters, Bernard (1881: 149) écrit:

«... lancer une colonne dans le Hoggar serait une folle entreprise qui, lors même qu'elle réussirait, n’aurait qu'un résultat infime et sans effet pour l'avenir. Le but à atteindre est plus facile et plus profitable ; c'est à In-Salah qu'il faut implanter la puissance française pour rendre la tranquillité au Sahara. Cette ville occupe une 
position centrale des plus remarquables : $|\ldots|$ Son marché, d une grande importance. est le centre d approvisionmement des Hoggar. Vivant du passage des caravanes qui viennent d'In-Salah. on pourrait, en occupant ce dernier point, les amener lacilement à composition."

De fait, en cette fin de siècle, les Kel-Ahaggar se retrouvent encerclés et surtout privés de leurs débouchés car, au sud comme au nord. leurs marchés traditionnels sont désormais aux mains des Français. Face à de telles menaces, l'amenakal, Ahitaghel ag Mohamed Biska. semble prôner une prudente politique d'attente et de réserve. Ainsi, désavouera-t-il toutes les attaques alors menées, notamment par les Taytoq". contre les habitants du Tidikelt. Sa politique était ainsi résumée par le général Servière (1"octobre 190()) : "Se tenir à l'écart en cherchant soigneusement à éviter tout conflit avec l'autorité française pour nous ôter tout prétexte d'intervenir dans les affaires du Hoggar » (AOM, 22H).

Dans cette situation critique, suite au décès, en 190(), d'Ahitaghel ag Mohamed Biska, une lutte serrée va mettre aux prises deux des prétendants à sa succession. Tous deux étaient des neveux utérins de l'amenâkal défunt. La stricte application des règles de transmission du pouvoir donnait priorité à Mokhammed ag Urzigh.

Mais, dans ce contexte politique difficile et surdéterminé par l'attitude à tenir face aux menées coloniales, les Kel-Ahaggar se retrouvèrent divisés en deux camps antagonistes. Une majorité d'entre eux, notamment parmi les tributaires, plébiscita Atici qui jouissait d'une réputation de guerrier valeureux et "qui personnifiait le parti hostile à la France » (Benhazera 1908: 126). Mais une partie significative des nobles opta, elle, pour Mokhamed ag Urzig. Cette situation provoqua l'intervention de religieux (ineslemen) désireux d'éviter un clivage préjudiciable au sein des KelAhaggar. Abidine, marabout Kunta particulièrement influent, choisit de neutraliser provisoirement ce conflit en prônant l'instauration d'un pouvoir bicéphale assuré conjointement par Mokhamed ag Urzig et Atici ag

5. Ce sont en effet les Taytoq qui mènent à cette époque la plupart des attaques ayant pour cible le Tidikelt, et ceci sans l'accord de leur chef (amghar) Sidi agg Akaraji alors réfugié che\% son oncle Ahitaghel et "depuis longtemps déjà impuissant à diriger sa tribu ". Suite à un contre-rezzou mené en juillet 190() par le Kebir Baba, ils quitterent l'Ahnet et. selon une lettre du chef de l'Annexe d'In-Salah (1.5 décembre 1900), essayèrent de trouver refuge dans l'Atakor " mais Ahitaghel craignant les inconvénients de leur voisinage les a invités à se retirer" (AOM, 22H). I es Taytog s installerent alors dans loued Afara en territoire Ajjer, et ce sans laaceord des Uraghen. Significativement. à celte époque, les Taytog sont considérés comme les principaux adversaires de la pénétration françatse, comme "la seule fraction qui depuis notre arrivée au Tidikelt nous ait lémoigné une hostilité bien caracterisée » (ibid.).

6. Ve serait-ce que paree que Mokhammed ag lirsigétait le fils aine de la premiere sceur cadelle (Katouh) d' Ahitaghel el Alici ag Amellat le tils ainé de la seconde seur cadetle (Khawila) d'Ahitaghel. 
Amellal7. Il s'ensuivra une situation de désordre et de confusion particulièrement préjudiciable aux tributaires qui se voient pressurés par des pouvoirs concurrents. On assiste alors à un véritable «éclatement » de la confédération des Kel-Ahaggar. Mokhamed ag Urzig fut rapidement éclipsé par Atici ${ }^{\ngtr}$ alors que d'autres nobles Kel-Ghela voient dans cette situation confuse leur autorité s'accroître. Tel est le cas de Mokhammed ag Ghotman, petit-fils de l'ancien amenâkal El Khadj Akhmed, dans la région de Tazeruk, et surtout de Mûsa agg Amastân qui, dans l'Adagh, rassemble autour de lui sa "clientèle »: Kel-Ahaggar las des divisions internes, désireux de pouvoir à nouveau commercer avec le Tidikelt et favorables à une politique de bonne entente, mais également Touaregs de l'Aïr (Kel-Tédélé) et Ifoghas de l'Adagh (Bourgeot 1995: 302).

Cet éclatement ne signifie d'ailleurs pas que des actions communes ne puissent être menées. Ainsi, fin 1901, un fort rezzou Kel-Ahaggar attaqua les Berabich de l'Azaouad soumis aux autorités françaises de Tombouctou (cf. Foucauld 1984 : 146). Suite à une demande des groupes Berabich non encore ralliés, cette expédition fut décidée par Atici après consultation des principaux notables Kel-Ahaggar parmi lesquels son frère Anaba, Sidi agg Akaraji et Mûsa agg Amastân. Tous ces hommes participeront d'ailleurs à ce fructueux rezzou sur lequel un rapport du lieutenant Cottenest (AOM, 22H) fournit de précicux renseignements.

\section{De Tit à In-Salah}

Dans ce contexte, Mokhammed ag Urzig, pour nuire à Atici dont l'autorité lui était supérieure, monta une véritable provocation qui eut pour conséquence l'arrivée des Français en Ahaggar'. Trois Kel-Ahaggar ayant à leur tête Baba ag Tamaklast attaquèrent et dévalisèrent de leurs biens Mohammed Ben Messis puis sa sceur Fatma. Cette agression, qui venait après bien d'autres exactions commises par les Kel-Ahaggar, mit en émoi

7. Geste symbolique: Abidine coupa en deux le voile de tête destiné à l'amenâkal et revêtit chacun des deux prétendants d'une des moitiés ainsi obtenues (BHNHAZERA 1908: 126).

8. Fait significatif: en décembre 1901, les autorités d'In-Salah apprennent et le décès d'Ahitaghel et son remplacement par Atici. Il n'est fait alors aucune mention de Mokhammed ag Urzig. Atici, quant à lui, est présenté comme bénéficiant chez les Kel-Ahaggar "d'une réputation très affirmée d'équité et de modération " (lettre du général Servière, 31 janvier 1901. AOM, 22H).

9. C'est du moins ainsi que fut très vite présentée cette attaque. Benhazera écrit que Baba ag Tamaklast était "poussé par Mokhammed ag Ourzig, désireux de causer des embarras à son rival heureux Atici... » (BENHAZI:RA 1908: 127). Quant à Laperrine, il estime clairement que «... derrière Ben Messis et sa sœur c'était nous qui étions visés" (LAPtRRINE 1913: 395). D'où il «... s'ensuit que Mokhamed ag Urzig est à l'origine de ce combat [Tit] et que, de facto, il a favorisé la conquête coloniale afin de nuire à son cousin Atici " (Bourgeor 1984: 489). 
nombre de personnes au Tidikelt. Surtout. les militaires d'In-Salah. en se gardant bien d’en prévenir leurs autorités hiérarchiques qu ils savaient opposées à ce type d'opération". purent trouver là un prétexte suffisant pour intervenir. Après avoir évoqué un engagement de non-agression envers les Kel-Ahaggar pris par le prédécesseur de Cauved à In-Salah. Laperrine précise quelques années plus tard: "Il fut convenu cependant avec le capitaine Cauvet que nous le respecterions pour le principe, mais qu’à la première agression. à la première insolence, nous reprendrions vigoureusement la manière forte, fermant les marchés du Tidikelt aux caravanes Targuies et lançant un contre-rezon qui irait jusque dans les campements des Hoggars » (Laperrine 1913: 394).

Le capitaine Cauvet commandant du poste d’In-Salah décida done d'envoyer un "contre-reyzou " en Ahaggar. Le lieutenant Cottenest, à la tête d'un groupe de volontaires dans lequel ne se trouvait aucun soldat français, parcourt la région durant plusieurs semaines" ${ }^{\prime \prime}$ Sur le chemin du retour, à Tit. la colonne est attaquée par un important groupe de Touaregs. Grâce notamment à leur puissance de feu et à leur organisation militaire. les hommes du lieutenant Cottenest emportent là une victoire décisive. Nombreux furent les Kel-Ahaggar qui trouvèrent la mort durant ce combat. Le groupe des tributaires Dag-Ghâli qui fournissait le plus grand nombre d"assaillants y fut particulièrement touché et y perdit son chef Mohammed ag Bessa' ${ }^{12}$. Mais, au-delà même de ces pertes humaines, c'est sur le plan

10. Le commandant Cauvet est sur ce point très clair: " D’ordres supérieurs il n"y en avait pas eu. J'avais pris toutes mes précautions pour qu'on ne men donne pas " (in Dexis 1991: 127). Même après la réussite du raid Cottenest la nombreuse correspondance émanant des autorités militaires ou civiles. tant d'Alger que de Paris. reste trés critique quant à la décision prise par le commandant Cauvet. Crispation hiérarchique, méconnaissance totale des réalités sahariennes. crainte (rétrospective) d'un désastre type Flatters en sont les éléments les plus récurrents. Quant à l’importance politique du combat de Tit. elle est très largement sous-estiméce voire niée (AOM. 22H49). Cette attitude sera d'ailleurs reconduite pour le contre-rezonu de (juillo-l ohan : voir notamment la lettre (du 12 novembre 1902) du général Caze qui demandait ni plus ni moins le remplatcement de Cauvet (AOM. 22H49).

11. Outre le rapport de Cottenest (1988). on pourra se reporter. concernant cette

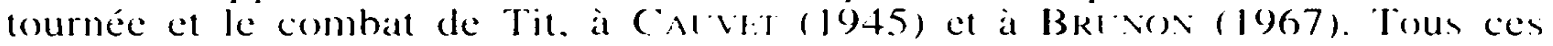
Écrits eependant présentent le point de vue des autorités françaises: aussi est-il heureux que (i. Barrere (1990) ait pu recueillir ches les Kel-Ahaggar celui des habilants d ldeles qui furent confrontés au passage de ce contre-ressou.

12. Selon Cautet: "Cottenest avait compté sur les lieux 71 tollareg morts sur place et les corps de 22 autres guerriers tués au cours de la poursuites soit en lout 9.3. De leur cóté. les Touareg accusatient seulement 61 morts. In réalité leurs pertes totales. dapres un relevé nominatif dressé par le pere de foucauld el comprenant ceux qui moururent par lat suite de leurs blessures, élevaient it plus de cent

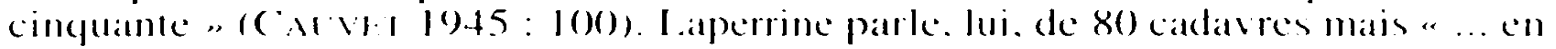
y comprenant les cadatres emportés et les blesses moth dans les deux ou trois

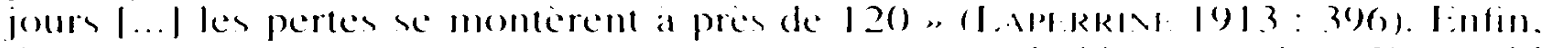
dams une mote accompagnant un texte en prose relatif all combat. Foncauld

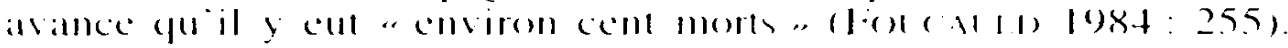


moral que les Kel-Ahaggar subirent le revers le plus important. Le combat de Tit, en mettant à bas le mythe de leur invincibilité, fut pour eux un vrai choc psychologique. Désormais leur territoire n'est plus inviolé et cette défaite l'ouvre de fait à l'avancée française.

Au lendemain de Tit, l'influence de Mûsa agg Amastân va aller grandissant parmi les Kel-Ahaggar ${ }^{13}$. Né en 1867, orphelin de père très jeune, Mûsa eut une enfance relativement délaissée qu'il passa pour l'essentiel à surveiller les troupeaux de ses proches. Adolescent, il se joignit aux nombreux rezzous qui s'en allaient opérer au sud et au sud-ouest de l'Ahaggar". Il s'y distingua très vite «... son intelligence, son courage, sa résolution, la sûreté de son coup d'œil et sa ténacité dans les entreprises le mirent en peu d'années au premier rang des chefs de bande... " (Foucauld 1925 : 367). Aussi brillant dans les expéditions guerrières que dans la vie galante propre aux Kel-Ahaggar, Mûsa devint très vite une des figures emblématiques de l'aristocratie touarègue. Mais, vers sa trentième année, une rupture importante se produisit dans sa vie. Rupture qui se marqua notamment par une religiosité accrue et qui fut due, semble-t-il, à l'influence prédominante de Bây al-Kunti. De son vrai nom Sidi Mohammad b. Sîdî 'Umar b. Sîdî al-Mukhtâr, Bây al-Kunti (1865-1929) était l'arrière-petit-fils de Sîdî al-Muktâr al-Kabîr al-Wâfî̀ (1729-1811) le fondateur de la puissance kunta. Parlant arabe et tamâhaq, Bây se trouvait à un point de confluence des traditions religieuses des Zwâya maures et des ineslemen touaregs (Norris 1975: 168).

Il résidait à Téleya dans l'Adagh, mais son l'influence était déterminante dans tout le Sahara et il fut pour Mûsa agg Amastân un véritable «maître spirituel ». Or, fidèle en cela à la politique de "collaboration » suivie par d'importantes confréries (Tidjaniya et Quadiriya notamment), Bây a toujours prôné une attitude pacifiste envers la pénétration française ${ }^{15}$,

13. Phénomène qui n’échappe pas aux autorités françaises. Dans une notice d'octobre 1902. Cauvet signale que Músa « paraît avoir remplacé l’amenoukhal Tissi à la tête du Hoggar». À la même époque, Laperrine note que Mûsa est le principal rival d'Atici qu' "il se pose en amenoukal des Hoggars depuis le contre-rezzou Cottenest... 》 (AOM, 22H49).

14. C'est durant cette période que Bellu, frère cadet de Mûsa, trouva la mort lors d’une expédition che\% les Iwllemmeden. Músa lui-même y fut sérieusement blessé. Quelque temps plus tard, il revint, à la tête d'un fort contingent de guerriers, venger la mort de son frère : ce fut là l'origine de la bataille d'lzerwan (NICOLAS 1950: 64; Bl:RNIS 1970: 463 et Al.AWJiLY 1975: 120-135). Ces épisodes guerriers sont évoqués dans de nombreuses poésies tant chez les KelAhaggar (Folicalti.) 1925-1930) que chez les Iwllemmeden (Nicolas 1944 et CAstei.l. GatTinara 1992).

15. Dès les tous débuts de leur installation au Sahara, les militaires français auront recours à l'influence excrcée par Bây sur les populations locales (cf. MARTY 1920, I : 116-122 et Richi:R 1924). Selon MétO1S (1995: 25), c'est sur les conseils de Bây que serait due pour une bonne part la demande de soumission des Ifoghas aux autorités d'In-Salah en 1904. On notera que la recherche de 
à la différence de son cousin Abidine al-Kunti qui s’en avéra. lui, durant toute sa vie, un adversaire résolu (cf. Aouad-Badoual 1993).

Quand en 1900 s'ouvre la succession d'Ahitaghel. Mûsa est déjà un des hommes qui comptent parmi les Kel-Ahaggar, et à ce titre il sera un des candidats en lice au rang d'amenukal's. De par sa place dans les lignées Kel-Ettebel, il ne peut se prévaloir d une quelconque priorité sur Mokhamed ag Urzig et Atici ${ }^{17}$. Mais il peut cependant mettre en avant sa qualité de neveu agnatique de Khyar ag Hegyer. Celui-ci, tout comme son frère Amastân. était issu d’un premier mariage de Tigent, la femme de Elkhaj Akhmed. Il fut «adopté » par ce dernier, vécut auprès de lui et participa au pouvoir à ses côtés. À la mort d’Elkhaj Akhmed, ne pouvant se prévaloir d'une place dans la ligne directe de transmission du pouvoir, il dut accepter la désignation d'Ahitaghel (neveu utérin d'Elkhaj Akhmed) comme amenakkal. Mais tout semble indiquer, selon les termes d'une lettre de Féraud en date du 13 mai 1881. qu'il «... s'est toujours considéré comme frustré de ses droits par l’élection d'Aitar'el, auquel il n'a cessé de faire de l'opposition ${ }^{1 x}$. Khyar sut faire profiter son neveu et de ses propres réseaux d'influence et de son habileté politique. La lutte pour le pouvoir qui va opposer Músa, neveu agnatique de Khyar et Atici, neveu utérin d Ahitaghel, reproduit celle qui une génération auparavant avait mis aux prises Khyar et Ahitaghel (Bourgeot 1984: 490).

Sur ce point. les militaires d'In-Salah sauront assez vite à quoi s'en tenir. Quand la colonne dirigée par Cottenest s'apprête à quitter In-Salah, quelques Dag-Ghâli se trouvent de passage dans cette oasis. Ils sont alors renvoyés dans l'A haggar chargés de lettres pour les principaux notables. Dans ces correspondances figurent deux points principaux. Le premier est lannonce que les marchés du Touat sont désormais fermés aux KelAhaggar. Le second est ainsi résumé par Cauvet : "... ils étaient prévenus que nous ne voulions plus avoir la moindre relation à l'avenir avec leur

l'appui des religieux semble avoir été un trait constant de la politique coloniale française tant dans le Sud algérien rutilisation des confréries: Tijániyya. Rahmaniyya et Qádiriyya) qua au Maroc (marabouts d'(Ouezoan).

16. Pour succéder à Ahitaghel, selon For (N)1.1) (1994-1995: 23), quatre KelAhaggar se retrouverent concurrents: Atici. Mokhamed ag Urzig. Sidi Mokammed ag (jhotman et Mûsa agg Amastân. Information confirmée par de nombreux rapports de lépoque (êf. Mi:rols : A(OM, 22H30).

17. Parmi les aristocrates Kel-(ihela ceux hénéficiant de l'ettehel, droit dacces au commandement transmis par voie matrilinéaire pouvaient prétendre devenir ame'nikal.

18. Selon LAPtRRIN: (19) 3: 4(07). Khyar "... atvait voté dans la djemáa pour que lon sopposát par la force all passage de flatlers mais il fut indigné des procédés de trabison employés par Atici. Altacyuer par surprise est un procédé de combat. mais engager sal parole avee un ennemi pour mieux le faire lomber dans l'embuscade. son hommeur ne ladmil pas. Par la suite. avec laarrivée des liançais dans l Ahaggar. Khyar eut de nombreax comtacts ated laperrine et selon les

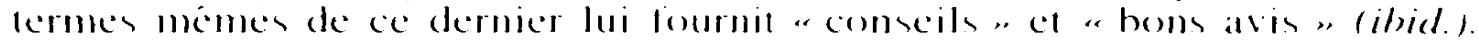


Amenoukal actuel et avec ceux de leurs notables qui avaient pris part au massacre de la Mission Flatters. Les seuls autorisés à entrer en relations avec nous étaient Khiar ag Adjier, homme honorable, mais déjà usé, et son neveu Moussa ag Amastane " (Cauvet 1945:41) ${ }^{19}$.

C'est dire qu'avant même le combat de Tit qui, bien évidemment, accentuera encore cette situation, deux hommes (Mûsa et Atici), et à travers eux deux lignes politiques divergentes, se dégagent au sein des Kel-Ahaggar. Les autorités coloniales feront dès lors tout pour exploiter à leur profit les contradictions internes du groupe dirigeant en jouant Mûsa contre Atici.

Dès août 1902, après maintes discussions et tractations avec ses pairs, Mûsa agg Amastân entre en contact avec les militaires français d'In-Salah pour demander la paix et le libre accès aux marchés du Tidikelt (AOM, 22H49). Les autorités françaises vont alors rapidement voir en lui un interlocuteur privilégié, la force sur laquelle il faut s'appuyer, et ce contre Atici et sa politique de résistance ${ }^{20}$. Elles chercheront dès lors à renforcer son influence chez les Kel-Ahaggar. Dans une lettre du 14 septembre 1902 (AOM, 22H49), le général Servière résume ainsi la politique suivie par ses subordonnés hiérarchiques dans le Sud algérien : "Le commandant Laperrine et le capitaine Cauvet ont intentionnellement fait répandre le bruit chez les Touaregs qu’il leur répugnait de traiter avé les assassins du colonel Flatters. La djemaa des Hoggar qui connaissait cette répugnance et qui voulait la paix a par suite écarté Atici qui était compromis dans

19. Considérer la participation au massacre de la mission flatters comme une pierre d'achoppement quant à l'attitude à tenir face aux Kel-Ahaggar est un theme récurrent de toute la littérature coloniale. On peut cependant se demander sil n'y a pas là une sorte de justification après coup de la politique suivie. Cauvet lui-même reconnaît qu'en 1902 "... on ne se rappelait plus très exactement ce qu'avait été la Mission Flatters. On savait seulement d'une maniere asse\% vague. que le colonel avait été surpris et tué par une harka de Touareg $|\ldots|$ mais on était loin de soupçonner le rôle personnel d'Attissi...". Quant à laperrine son opinion est clairement énoncéc dáns une lettre adressée le 7 avril 1902 à Cauvet : "Pour la mission Flatters c'est de la bien vieille histoire et pour mon compte j'ai pris 16 ou 17 fusils en provenance $[$ sic/ et fait couper les têtes de gens pris dans cette affaire il y a 6 ans déjà en 1896. Pour mon petit bout /sic!/ cela serait une vengeance suffisante... " (AOM, 14X2).

20. Mais cela n'a pas été aussi simple que le laisse penser la reconstruction historique de ces événements. Ainsi le commandant Cauvet n`a pas cru, dans un premier temps, à la sincérité des propositions de Mûsa agg Amastân au contraire de Laperrine qui, fort de son expérience chez les Touaregs de la région de Tombouctou, écrivait (le 2 octobre 1902) à son subordonné hiérarchique: "Je crois que tu fais fausse route au sujet de Moussa ag Amastane. Les chefs Kountah de Tombouctou le représentaient comme un homme de parole ; comme Chaboun des Tengueriguef $|\ldots|$. Chez les Touaregs le chef n'est pas absolu, il a besoin de palabrer, denvoyer des émissaires dans les divers campements... etc. Sa lettre me paraît sincère et c'est malheureux que la chose ait été brusquement rompuc. [...] Pour le moment il faut boucler sérieusement les marchés $|\ldots|$ et le mieux serait de reprendre avec Mousa si la chose se peut » (AOM, 14X2). 
ce meurtre et a mis en avant son rival Moussa Agamastane. ce qui facilitera a l'avenir les négociations »?'.

Dans celte optique, les militaires français, dont la connaissance du terrain s"approfondit chaque jour, vont habilement utiliser les religieux du Tidikelt. Ces derniers, et notamment El Hadj Bilou pour les Ahl Azzi et Si Moulay Omar pour les Chorfa, ne sont pas sans influence chez les KelAhaggar. De plus les rivalités qui les opposent de longue date 22 créent entre eux une compétition dont l'autorité coloniale saura tirer profit. Dans une lettre en date du 2 décembre 1903 (AOM, 14X2), Laperrine écrit à Cauvet: "Au Tidikelt il y a lutte acharnée entre Billou et Moulay Omar. Si Métois sait en tirer parti cela ira tout seul, cest à qui réussira le micux. Billou veut Moussa. Moulay relance les Ifoghas, cest la course. » En 19(03. El Hadj Billou, à la demande de Mûsa agg Amastân et avec l'accord du capitaine Métois. se rend dans l'Ahaggar. But avoué de cette mission : user de son influence auprès des Kel-Ahaggar pour les rallier à Músa et surtout les convaincre que celui-ci doit se rendre, sans crainte aucune, à In-Salah pour y rencontrer les autorités françaises et signer avec eux un traité de paix ${ }^{23}$. Parallèlement à ces manoeuvres diplomatiques. d'importantes tournées menées durant les années 1902-1903 par les militaires français d'In-Salah apparaissent comme autant de démonstrations de force 24 :

- Doctobre à décembre 1902, un contre-rezzou de 150 méharistes. sous le commandement du lieutenant Guillo-Lohan, visite les principaux centres de l'Ahaggar et pour la première fois en traverse le coeur: l'Atakor.

21. Opinion qui se retrouve fréquemment dans les documents de l'époque. Ainsi dans une correspondance du gouvernement général en date du 28 février 1903. on peut lire : "Le chef de I'Annexe d'In-Salah avait très habilement fait répandre le bruit qu'jl ne consentirait jamais à traiter avec les assassins du colonel Flatters. Cette attitude avait eu pour résultat d'augmenter parmi les Hoggar l'influence de Moussa ag Amastane... " (AOM. 22H30).

22. Sur ces oppositions entre Ahl Axzi et Chorfa d'un cóté. et entre Ahl Azri è Kunta de l'autre. voir Botrobitor (1995: 294-296).

23. Voir la maniere dont Hadj Bilou, dans son rapport de tournée, présente ces événements : "Dans les premiers jours du mois de mai lo03. Ie nommé (chernach. hartani de Moussa ag Amastan arrivait à In-Salah portant de la part de son maitre plusieurs lettres |...l. Dans celle qui métait adressée. Moussa me disait qu'il desirait se reneontrer avee moi dans le but de faire la paix " (AOM, 22H30). Version confirmée par le capitane Métois qui précise que Músa lui avait demandé " de lui envoyer le caidd $\mathrm{bl}$ Hadj Ahmed hen Mohammed Bilou. qui. en sa qualité d'Ahl $A x, \mathrm{i}$. était susceptible d’inspirer confiance aux Touareg. Moussal savait hien qu il pouvait avoir contiance dans les français. mais il etait utile de le faire comprendre at eous les siems avant de lave une démarche décisive.

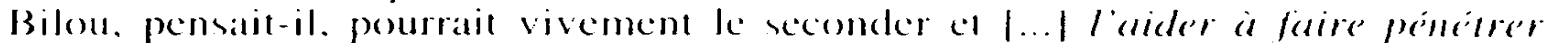

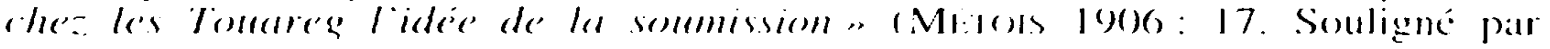
mo(is).

24. lilles sont atusi les premiers signes de la réorganisation. sous la direction de

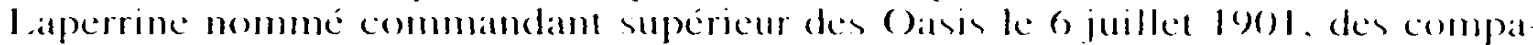

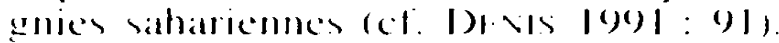


- Après une tournée qui lui permet, en janvier-mars 1903 , de reconnaître le Mouydir et la Tefedest, le lieutenant Besset atteint les campements des partisans d'Atici installés au sud-est d'Amguid (juin 1903).

- En avril 1903, le commandant Laperrine entreprend une grande tournée qui, par le Mouydir et l'Ahnet, le conduit jusqu'à In-Ziza.

Outre leur intérêt quant à la connaissance du terrain, ces tournées, dans la foulée du combat de Tit, viennent confirmer aux Kel-Ahaggar que leur territoire n'est plus inviolable. C'est là très certainement un nouveau choc psychologique. À cet égard, le contre-rezzou du lieutenant GuilloLohan qui peut, sans tirer un coup de feu, parcourir le sanctuaire de l'Atakor est exemplaire. De plus ces tournées qui, dès 1903, traversent la plupart des régions de l'Ahaggar permettent la mise en oeuvre des directives de Laperrine: politique de fermeté envers les opposants et d' "apprivoisement » avec les populations ralliées ou restées dans l'expectative. Enfin, en se montrant capable d'atteindre (en plein été !) nombre de campements ralliés à Atici, le lieutenant Besset porte un sérieux coup au moral des Kel-Ahaggar ${ }^{25}$. Fait significatif : dans la continuation de cette tournée, plusieurs groupes Isaqqamâren (Kel-Tefedest, Kel-Amguid, KelIngher notamment), jusqualors ralliés à Atici et l'ayant suivi dans son déplacement vers l’est, reviennent occuper leurs territoires habituels et se placent sous l'autorité de Mûsa ag̣g Amastân (AOM, 22H49).

\section{In-Salah, 1904}

En janvier 19(14, Mûsa agg Amastân, à la tête d’une importante délégation de Kel-Ahaggar, vient demander la paix à In-Salah. Offre aceeptée par le capitaine Métois qui le reconnaît comme chef de l'Ahaggar et le revêt du burnous rouge de caïd. Dès lors, "l'administration militaire française commence à intervenir directement dans la politique des Kel-Ahaggar avant même de s'installer dans le pays " (Gast 1986: 289). En cela, la rencontre d'In-Salah fut déterminante car elle est bien l'acte initial du ralliement de Mûsa aux autorités coloniales. Cependant, si son voyage à In-Salah marque incontestablement un tournant capital, il est loin de régler définitivement les problèmes en suspens. La base même sur laquelle se déroulent ces entrevues entre le capitaine Métois et Mûsa n'est d’ailleurs pas exempte d’ambiguïté. Dans une correspondance en date du 17 février 1904, Laperrine le reconnaît lui-même : on ne peut parler d'une véritable soumission des Kel-Ahaggar. Lors de ces entrevues d'In-Salah, le capitaine

25. Dans son rapport (AOM, 22H49), cet officier résume ainsi la portée politique de sa tournée: "La désillusion a été cruelle. Les dissidents Hoggar el Taitoq qui forment les partisans d'Atici et campent avec lui ne savent plus en quelle région aller chercher un asile. loin de nos coups. Ils commencent à comprendre les inconvénients de leur isolement. |... Effet moral qui laisse ces tribus sous le coup d'une attaque toujours possible alors qu elles se croyaient inaccessibles. et diminue d'autant l'influence d'Atici et du parti de la guerre...". 
Métois remit un texte contenant ses «Instructions» à Mûsa. Voici comment il présenta, deux ans après. cet épisode : "... nos négociations furent faciles. Je rédigeai sous la forme d'instructions à l'amenákal des Touareg, titre que je reconnaissais à Moussa, les exigences du gouvernement français. Moussa les accepta entièrement et ne me demanda que deux modifications, qui, toutes deux, constituaient une aggravation du document, au point de vue targui » (Métois 1995: 33) 26.

Ce document, consultable aux Archives d'Outre-Mer d'Aix-en-Provence $(A O M, 22 \mathrm{H} 3())$, porte le titre suivant: "Instructions délivrées par le Capitaine Métois. Chef de l’annexe d'In-Salah, au nom du Gouvernement Français, à Moussa ag Amastan, aménokal du Ahaggar, représentant de toutes les tribus nobles et imrad du Ahaggar, et lui faisant connaitre les conditions dans lesquelles le Gouvernement Français accepte sa collaboration. " Sa valeur historique est indéniable : il fixe le cadre politique dans lequel se sont déroulées et les entrevues d’In-Salah et les négociations postérieures. Or, à notre connaissance, ce texte, auquel nombre d'auteurs ont fait référence, n’a jamais été reproduit. Aussi le reproduisons-nous. ci-dessous, dans son intégralité.

"1) Moussa ag Amastan exercera le commandement de tout le territoire de l'Ahaggar et de ses dépendances. Il y assurera la liberté et la sécurité des voyageurs et des commerçants venus de la France ou des pays qui lui sont soumis. Un esclave portant de l'or sur la tête pourra traverser le Ahaggar en toute sécurité 27 .

2) Moussa ag Amastan recevra un burnous d'investiture et un cachet, comme les caïds des régions soumises, mais il conservera son titre d'aménokal el exercera le commandement. avec le concours de la djemaá, suivant les usages de son pays. 3) I e chef d'annexe d'In-Salah sera son intermédiaire. tant pour lui transmettre les ordres du Gouvernement Français que pour transmettre à celui-ci les demandes formulées par Moussa ag Amastan. soit en son nom propre, soit au nom de ses gens.

4) Moussa ag Amastan remettra au chef d'annexe d'In-Salah, lá liste complète des tribus, fractions ou sous-fractions placées sous son commandement, et des campements qu elles occupent habituellement ${ }^{2 x}$. Dans tout le territoire ainsi déterminé. et pour toutes les fractions ainsi dénommécs, les troupes du Gouvernement Français ne feront pas acte d’hostilité. Les habitants pourront en conséquence s'approcher des camps militaires. où ils seront reçus amicalement, et vendre aux soldats les produits dont ils disposent.

5) Toute infraction aux stipulations de larticle précédent sera signalée par Moussa ag Amastan au chef dannexe si elle a été commise par un militaire. et par le chef d"annexe à Moussa ag Amastan si elle a été commise par Moussa. Le coupable sera puni.

6) I Dans le cas où un homme des Imouhar méconnaitrait l'autorité de Moussa ag Amastan. celui-ci le signalerait au chef d'annexe qui pourrait alors le châtier.

26. Músa aurat fait ajouter la derniere phrase du point n" 1 ainsi que la derniere instruction (point n" $\left.n^{\prime \prime}\right)$ qui envisage la création d'un village a Tadjemut.

27. A cet endroit du texte une astérisque renvoie a la note suivante : Phrase ajoutée au texte primitif sur la demande expresse de Moussat."

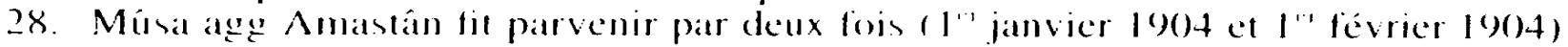
des decuments précisant tour ées points all capitatine Métois (voir A6)M. 221130). 
7) D’une manière générale, Moussa ag Amastan exercera lui-même, et avec le concours de ses gens, la police de son territoire. Dans le cas où il aurait affaire à un ennemi qui lui serait supéricur en nombre, il en informerait le chef d'annexe qui le seconderait au moyen des troupes dont il dispose.

8) Afin de bien affirmer aux yeux de tous la collaboration étroite qui existe entre le Chef de l'annexe d'In-Salah et Moussa ag Amastan pour la protection du commerce et le maintien de la paix, les gens qui serviront Moussa ag Amastan exécuteront ses ordres de police et lui serviront d'intermédiaires avec le Chef d’annexe, recevront du Gouvernement Français une indemnité mensuelle de 15 francs. Leur nombre sera fixé en raison des crédits accordés par le Gouvernement Français, et le Chef d'annexe pourra, pour récompenser ceux d'entre eux qui se seront plus particulièrement fait remarquer par leur zèle, leur confier un fusil de l'État, avec les munitions qu'il comporte.

9) Dans les territoires voisins du Ahaggar et actuellement soumis à la France, les Imouhar seront reçus amicalement, et devront s'abstenir de tout acte d'hostilité. Le Chef d'annexe fera connaître à Moussa ag Amastan les modifications qui pourraient être apportées à la situation politique du pays; de même qu'il fera connaître à ses collègues de Tombouctou, de Gogo, de Thaoua et de Zinder que le Ahaggar est maintenant en paix avec la France, et que ses habitants doivent être reçus partout en conséquence.

10) Afin de faciliter les relations entre le Ahaggar et le Tidikelt, le Chef d'annexe d'In-Salah s'occupera de la création d'un village à Tadjemout où des harratin du Ahaggar pourront cultiver et où des travaux seront exécutés pour l'aménagement des cultures. "

Ce document est porteur d'un certain nombre d'ambiguïtés. Mûsa agg Amastân y est présenté comme amenâkal des Kel-Ahaggar alors qu'à cette date il ne peut se prévaloir d'un titre dont les siens ne l'avaient pas investi. Le seul amenûkal «élu » par les Kel-Ahaggar est toujours Atici ag Amellal et, à cette date, ce dernier représente encore une force politique importante dans l'A haggar.

Bien que politiquement dépendant des autorités coloniales, on semble reconnaître à Mûsa (point $\mathrm{n}^{\circ} 2$ ) une autonomie importante ${ }^{24}$. Surtout, il n'est à aucun moment fait explicitement mention d'une soumission des Kel-Ahaggar. Fait significatif : il est soigneusement évité toute référence à un quelconque impôt, même symbolique, comme à toute possibilité d'installation française dans l'Ahaggar. La mise en avant principale restant celle de la liberté de circuler et de commercer (points $n^{\circ} 1,4$ et 9 ). Le capitaine Métois était d'ailleurs parfaitement conscient de ces ambiguïtés. Quand le 10 février 1904 il expédie une copie de ces «Instructions » à Laperrine, il précise dans une lettre accompagnant son envoi :

"Sans doute, on aurait pu obtenir dans les conditions actuelles, une soumission plus étroite que celle que font ressortir les instructions ci-jointes. Mais pour

29. Dans une lettre à Laperrine (10 février 1904), rendant compte de ses entrevues avec Mûsa, le capitaine Métois écrit : « $J$ 'ai alors ajouté que pour tout le reste, le Gouvernement Français ne désirait que le bien des Imouhar comme de tous ses autres sujets, et qu'il les laisserait s'administrer intérieurement à leur guise » (AOM, 22H30. Souligné par nous). 
demander une soumission plus étroite il fallate avoir leintention de donner à lous les actes hostiles qui pourratent encore se produire une sanction. Il the mia pas semblé que lelles fussent actuellement les intentions gouvernementales.

Jai done cherché une solution provisoire, mais d'un provisoire capable de durer atussi longtemps que les incertitudes sur ee quon veut faire du Sahara.

|... $\mid$ On a vu ailleurs quels résultats pouvaient parfois amener une précipitation trop grande et les Touareg constituent certainement une des populations les plus méfiantes, pour ne pas dire la plus métiante. Pour ne pas enlever toute influence a son chel actuel. il faudra sans doute au début une grande modération dans la protection que nous lui accordons.

Telles sont les idées dont je me suis inspiré dans cette circonstance. Je les crois justes en ce moment. sans me dissimuler qu elles cesseraient de létre le jour ou on ne reculerait pas devant l'oceupation du Ahaggar » (AOM. 22H30).

Politique prudente. justifiée tant par le caractère attribué aux KelAhaggar que par la situation politique régnante. Si linfluence de Mûsa semble de plus en plus importante. Atici et ses partisans sont loin d'être réduits à l'impuissance et toute erreur pourrait voir leur camp et la ligne politique qu'ils représentent reprendre le dessus" ${ }^{30}$. Ce n'est pas là le seul flou des négociations d’In-Salah. Bien d’autres questions n`y ont pas reçu une réponse définitive: attitude des autorités coloniales par rapport à la personne même d'Atici, problème du rattachement des Ifoghas, relations entre Kel-Ghela et Taytoq et entre Kel-Ahaggar et Kel-Ajjer... Autant de problèmes auxquels seront bientôt confrontées les autorités d'In-Salah et notamment le capitaine Dinaux lors de son importante tournée de 1905.

\section{Métois/Laperrine}

Mûsa agg Amastân arriva à In-Salah le 21 janvier 1904. Dans une lettre au capitaine Cauvet, son prédécesseur à In-Salah. Métois précise : «Il y a une dizaine de jours que Moussa est arrivé accompagné de deux autres nobles et d'une suite de 70hommes représentant toutes les tribus du Hoggar 》 (in Lehuraux 197(): 31). Mûsa resta à In-Salah jusqu au 14 février 1904. Durant son séjour. la personne la plus haut placéc qu il y rencontra fut le capitaine Métois, chef de l'Annexe d'In-Salah.

Laperrine, qui se trouvait alors à Adrar, ne vint pas à In-Salah rencontrer le chef touareg. Dans plusieurs lettres privées adressées à (auvet (AOM. 14X2), Laperrine sexplique sur ce fait: "Moussa est à In-Salah. quelques jours plus lót il my trouvait mais je n'ai pas voulu avoir lair d'aller le voir. lorsqu`il ne se dérange pas pour me voir moi » (in lettre du 3.2.1904). "Moussa a passé prés d'un mois à In-Salah, javais bien

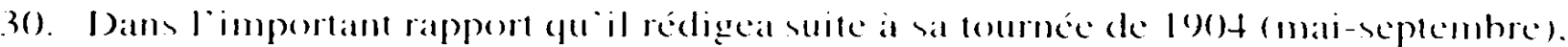

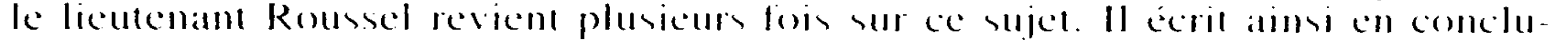
sion: "IDes renseignements que jaj recueillis el de ce que jai pu comstater

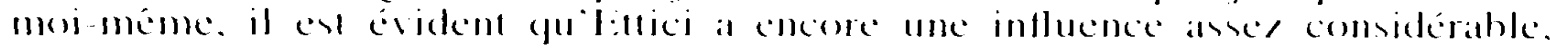

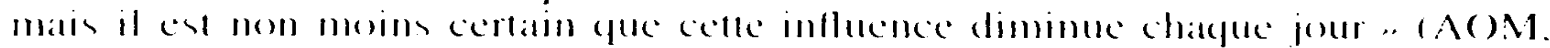
2216071 
envie d'y aller, mais j'aurais eu l'air d'aller lui présenter mes hommages » (in lettre du 25.2.1904).

Or, cette position plusieurs fois affirmée ${ }^{31}$ n'est en rien anecdotique. Elle est un signe parmi d autres des divergences politiques qui se révèlent, dès cette époque, entre Laperrine et Métois. Quelques mois plus tard ces divergences seront encore plus manifestes et se transformeront en un véritable conflit. Laperrine affirmera alors que si Mûsa n’a pas poussé jusqu'à Adrar, en février 1904, c'est qu'il en fut empêché par Métois: «...j'ai su que c'est déjà lui [Métois] qui l’a empêché [Mûsa] d'aller à Adrar comme il voulait le faire » (in lettre à Cauvet, 18.6.1904).

Reste à cerner le pourquoi et le comment de cette opposition entre deux hommes qui jouaient alors un rôle fondamental dans la politique mise en ceuvre envers les Kel-Ahaggar. Outre de possibles incompatibilités personnelles, tous deux se rattachent manifestement à des univers idéologiques opposés. Le capitaine Métois, quand il entra aux Affaires indigènes en 1902, était précédé d'une double réputation. Celle d'un brillant officier qui a participé à la mission Foureau-Lamy, mais aussi celle d'un franc-tireur qui dans un milieu militaire fortement marqué par un catholicisme traditionnel affirme haut et fort ses convictions républicaines et a-religieuses, voire anticléricales. Ainsi, fait significatif, quand I aperrine décida d’emmener avec lui le père de Foucauld lors de sa tournée de mars-juin 19(4), il se montra très inquiet des possibles incompréhensions qui pourraient surgir entre les deux hommes ${ }^{32}$.

31. Ainsi dans une autre lettre à Cauvet, en date du 10 février 1904, Laperrine explique: "Moussa ne veux /sic/ plus quitter In-Salah, il se prolonge et me fait retarder ma tournée car je ne veux pas avoir l'air de lui rendre visite" (AOM, 14X2).

32. A tort d'ailleurs. Laperrine ignorait, semble-t-il, que Foucauld et Métois avaient déjà éu loccasion de se rencontrer en février-mars 1903 à Beni-Abbès. Malgré leurs opinions philosophiques et religieuses opposées, lientrevue fut très cordiale si l'on se réfèrè à un article postérieur de Métois, paru dans le journal La (iriffe (1933) : « [... je n’étais pas fâché de recueillir l'avis de Charles de Foucauld. Aussi mon premier moment de liberté, en arrivant à Beni-Abbès, lui fut-il consacré. Je me rendis donc à son ermitage et il me reçut avec la plus grande cordialité. Pour éviter tout malentendu entre nous, je crus l'avertir de mon état d'esprit quant aux croyances catholiques et préciser que mon hommage n'allait pas au moine. mais à l'éminent explorateur du Maroc. au Maître en exploration à qui le modeste élève inconnu que j'étais venait demander une leçon. Charles de Foucauld ne se formalisa nullement de ma profession de foi a-religieuse. Il me prouva d’ailleurs qu’il n'était pas aussi retiré de la vie publique qu aurait pu le faire croire son existence solitaire... Il était parfaitement au courant de ce qui se passait dans le monde musulman, et méme mes communications à la Société de Géographie d'Alger ne lui avaient pas échappé. Aussi s'était-il réjoui de ma désignation pour le commandement d'In-Salah... » (in IDE SURIMAIN 1994 : 21). De son côté, Foucauld avait su apprécier à sa juste valeur le capitaine Métois comme l'indiquent quelques notations du Carnet de Beni-Abbès (Fol: CALLD 1993: 130) el surtout des extraits de lettres inédites citées par $M$. de Suremain (1994). 
Il est également vraisemblable que, vu leurs ambitions, une véritable compétition existait entre Laperrine et Métois. Ce dernier. du fait même de sa participation à la mission Foureau-Iamy, n’était pas un obscur officier saharien. Sur un point au moins, Laperrine et Métois pouvaient se trouver en concurrence : qui de ces deux hommes réussirait le premier la jonction entre les forces "algériennes » et les forces "soudanaises»? I.on sait que ce fut là le grand rêve de Laperrine. Il ne put le réaliser en 1904 quand la tournée à la tête de laquelle il se trouvait fut contrainte par le capitaine Théveniaut de faire demi-tour à Timiaouine le 16 avril $1904^{33}$. Mais, trois ans auparavant, dautres militaires avaient caressé le même projet. Fin octobre 1901, une commission inter-ministérielle décidait qu’une mission partirait de Gao pour rejoindre In-Salah. Trajet proposé : Gao-Adagh oriental-Tin Missao-In Zize-In-Salah. La dernière étape était considérée comme la plus dangereuse.

Le capitaine Reibell, qui faisait partic de cette commission, proposa qu'une deuxième mission, partie, elle, d’In-Salah, vienne à la rencontre de la colonne soudanaise. Sous couvert de sécurité, Reibbel visait deux objectifs bien plus importants. Il s'agissait d'abord de ne pas laisser aux seuls "soudanais" le bénéfice d'une telle opération. Enfin et surtout, les officiers algériens pourraient ainsi « prendre possession » de territoires qui, situés au sud d'In-Salah. n’étaient pas encore devenus des terres françaises. Dans une correspondance adressée à Métois le 3 novembre 1901, Reibell écrit :

"La seconde partie de l'entreprise d'Inzize à In-Salah me paraît risquée si l'Algérie ne leur tend pas la main. En envoyant à $M$. Révoil le compte rendu de la séance de la Commission à laquelle il nassistait pas, je lui ai proposé denvoyer au Hoggar une mission d'exploration. d'étude et de prise de possession |...l. I.e but serait de prendre pied au Hoggar et de tendre de là la main aux jeunes présomptueux venus du Soudan. L'Algérie doit avoir honneur à ne pas abandonner à d'autres la tâche qui lui incombe, 3.4 .

Mais, dans ses propositions à P. Révoil. alors gouverneur général de l'Algérie, Reibbel ne se contente pas de fixer les objectifs de cette opération. il s’attarde aussi sur les détails pratiques (organisation et composition de la colonne par exemple) et propose notamment que le colonel Pein et Métois soient les deux chefs de cette mission. Rien d'étonnant à cela: ces deux hommes avaient, tout comme Reibell, pris part à lat mission

33. In empêchant laperrine de mener sa mission jusqu à Tombouctou. Théveniaut ne faisait quebéir aux ordres du gouverneur général de l'Aol:. eux-mêmes approusés par le conscil des ministres. (el épinode célebre at souvent été commenté du seul point de vue "algérien " parr une littérature glorifiant laperrine "t le pere de foncaulak. Pour une vue plus "équilibrée " de cel événement. voir

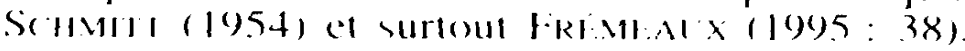

34. Nous temons at remereier M. de Suremain d avoir been voulu attirer motre attention sur cel important decument. 
Foureau-Lamy et tout indique qu'ils entretenaient depuis des relations amicales $^{35}$. Mais il est bien évident aussi que la proposition de Reibell laisse, par contre, Laperrine sur la touche. Dans sa lettre à Métois, Reibell ne s'en cache pas. Le ton quelque peu condescendant avec lequel il évoque le sort de Laperrine suggère bien que, pour le moins, de sourdes luttes d'influence agitaient alors le milieu des officiers sahariens.

«Les milices sahariennes et leur chef, le Comt. Laperrine, seraient laissés à leur ceuvre d'organisation des territoires des oasis sahariennes, rôle moins brillant que celui de la Mission Pein-Métois, mais plus utile peut-être et en tout cas il ne faut pas mêler les rôles. [...] Voyez donc le $\mathrm{Ct}$. Lévé et soyez éloquent. Il est facile à persuader. Insistez sur l'avantage et la nécessité de séparer nettement la tâche des Missions sahariennes destinées à une occupation méthodique, permanente des Oasis de celles d'une Mission d'exploration. On ne peut être à la fois au four et au moulin. Laissez. le Ct. Laperrine à la tâche délicate, ingrate et nécessaire qu'il a acceptée. Utilisez les aptitudes, les connaissances, l'expérience de Pein et de Métois à faire une ouvre également utile et nécessaire sur un autre théâtre d'opérations. Voilà qui vaudrait mieux pour vous que les oasis sahariennes. »

Certes, cette tentative échouera et, en 1904, les rôles prévus par Reibell seront en quelque sorte inversés. Métois se retrouvera plus ou moins cantonné à In-Salah alors que Laperrine pourra, lui, tenter de rejoindre Tombouctou. Mais il est peu vraisemblable qu'en 1901 Laperrine n'ait pas été averti de ce projet et de ses arrière-pensées ${ }^{36}$. Il est probable également qu'il en ait gardé quelque rancoxur envers Métois dont les liens d'amitié avec Reibell étaient alors largement connus.

Cependant, cette opposition entre Métois et Laperrine ne peut s'expliquer par ces seules divergences idéologiques ou par ces rivalités d'ambition. Plus importante nous paraît en effet l'opposition politique qui très tôt se manifeste entre ces deux hommes au sujet de la politique à conduire envers les populations du Sahara central. Bien des documents nous manquent encore sur ce point, mais on peut cependant tenter de cerner quelques-uns des principaux moments où cette opposition se manifesta.

35. Disposant d'importants moyens militaires et logistiques, cette mission réalisa la première traversée du Sahara. Partic le 23 novembre 1898 de Sedrata (au sud d'Ouargla), elle atteignit Agadez le 18 juillet 1899 et Zinder le 2 novembre 1899 (voir Fol:Rt:Al: 1902. ReIBEIL 1931 et POTTHtR 1951). Métois, comme Reibell, y participa de bout en bout. À la tête d'un goum levé à Ouargla, Pein, quant à lui, assura la protection de la mission lors de son passage dans l'extrême Sud algérien (LfHHURAl: 1935 ).

36. Le 15 août 1903, dans une lettre au colonel Pein, Laperrine évoque de manière très critique un important projet de colonne transsaharienne dont la composition n'est pas sans rappeler celle proposée par Reibell. Cette lettre figure dans le livre de Lehuraux consacré au colonel Pein (Lehuraux 1935: 142). Elle y est accompagnée de la note suivante dans laquelle Lehuraux cite des propos de Cauvet : "La colonne transsaharienne dont parlait Laperrine avait en réalité pour but de l'évincer, lui, des oasis où d'aucuns voulaient le faire remplacer par R... Je ne crois pas que Laperrine se soit douté de cette combinaison qui était bien visible. »Il est fort possible que ce R... ne soit autre que Reibell. 
Dès les tous débuts de Métois à In-Salah. des désaccords se maniféstèrent entre les deux hommes. notamment en ee qui concerne le choix d'un chef pour le groupe des Touaregs de l'ouest ${ }^{37}$. Métois proposait de sappuyer sur Badjeloud, chef (amghar) des Kel-Ahnet, qui, le premier parmi les Kel-Ahaggar, s'était rendu à In-Salah pour y signifier la soumission des siens aux autorités françaises. Laperrine, quant a lui. porta son choix sur un noble Taytog: Aziouel ag Seghada. Cette divergence de vue est ouvertement et longuement évoquée par Métois dans son livre de 1906. Il y écrit notamment :

"Au cours de sa tournée dans l'Ahenet. le commandant Laperrine avait organisé le pays. dont la soumission était un fait accompli depuis quelque temps déjà. Badjeloud était venu à In-Salah faire acte de soumission, et à ce titre méritait quelques égards: mais le commandant ne l'avait pas trouvé assè décoratif et avait confié le pouvoir à Aziouel ag Seghada. Sans doute. Aziouel était noble. et Badjeloud ne l'était pas. |... Quelle que fût mon opinion sur le choix d'Aziouel. comme amghar, je n"avais qu’à m’incliner devant sa désignation " (Métois 1995: $(7-2.3)^{3 x}$.

Ainsi, selon Métois, c’est parce que noble (et plus " décoratif "!) que Laperrine a préféré Aziouel à Badjeloud. Il y a là un signe parmi d'autres d'une des divergences existant entre Métois et Laperrine. Le premier propose de ne pas tenir compte de la hiérarchisation propre à la société touarègue, voire, quand cela est possible, de tenter de la détruire. Le second estime politiquement plus rentable, sinon de respecter, du moins de ne pas bouleverser (dans un premier temps au moins), cet ordre hiérarchique. Mais il est également évident que sous la plume de Métois ce passage révèle une autre critique: celle de la communion idéologique existant entre un Laperrine et les nobles Kel-Ahaggar. Et cela, même si en la personne d'Aziouel se " réalise le type du Targui suivant une certaine école: menteur, pillard ét traître. C'est l'homme du beau coup de sabre et de toutes les sottises $\gg$ (ibid.: 18).

Mais c'est bien suite aux entrevues d'In-Salah que ces divergences de vue se transformèrent en conflit plus ou moins ouvert. Que reprochent Iaperrine (et les officiers qui l’approuvent) à Métois? La divergence principale porte, semble-t-il, sur la politique à suivre envers les KelAhaggar: doit-on, après le combat de Tit, les traiter en vaincus devant - de gré ou de force -.. se soumettre corps et biens à la France, ou peuton, du moins dans un premier temps. considérer Músa comme le représentant d’un groupe défait militairement mais gardant néanmoins une

37. Insemble constitué par les nobles Taytog el par les groupes tributatires qui keur sont ratlachés: Kel-Ahnet et Tégehe-n-tifis principalement.

38. 1) atutres raisons intervenatent dans le peu de sympathic quéprousail Métois

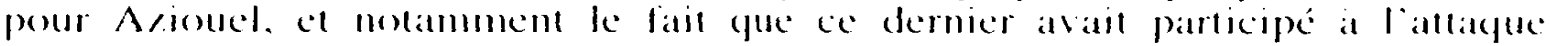

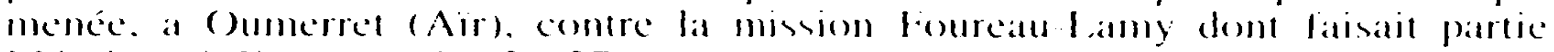
Mélois (ct. Fir) Rl 19)2: $27(1)$. 
autonomie politique? Les points d'achoppement entre Métois et laperrine sont à cet égard significatif's :

- La forme même des entrevues d'In-Salah. Il semble, pour reprendre les termes mêmes de Dinaux, que la réception de Mûsa à In-Salah fut «brillante ». Dans une lettre où il déplore le peu de crédits affectés au Tidikelt, Laperrine rapporte à Cauvet que Métois « ... a fait à Moussa une réception de $4800 \mathrm{~F}$ ! » (in lettre du 4.10.1904). Significativement dans son ouvrage sur La soumission des Touareg du Nord. Métois revient sur ce point et essaye manifestement de se justifier: "Il |Mûsal arrivait avec une allure de chien fouetté qui justifiait de ma part toutes les exigences. Je me montrai magnanime. J'envoyai au devant de lui l'officier interprète Durand, avec une escorte de cavaliers et de méharistes, pour lui souhaiter la bienvenue. L'empressement de la population d'In-Salah, qui tout entière formait la haie, sans que l'ordre en eût été donné, montrait, d'autre part, l'importance que cet événement avait pour la région. »

Bien évidemment ces critiques, plus ou moins voilées, contre la forme même qu'a prise la réception d'In-Salah, vont bien au-delà d'un simple problème d'utilisation des fonds publics. Ce qui est en jeu n'est ni plus ni moins que le statut même de Mûsa et l'appréhension politique de sa venue à In-Salah. C'est dans une lettre que Dinaux (alors chef de l'Annexe d'In-Salah) adresse à Laperrine qu'apparaît le plus nettement cet enjeu ${ }^{3 \prime}$. Dans cette correspondance, en date du 15 mars 1905 , Dinaux annonce qu'il vient de recevoir Sidi ag Keradji, "chef incontesté des Taytoq ", à In-Salah. Il précise: "Je me suis attaché dès le début à éviter toute démonstration extérieure telle que convocation de Caïds, de goums et nanbbas. "

Première raison invoquée pour justifier cette absence de faste : marquer nettement une différence dans le traitement accordé à Mûsa ( venu en ambassadeur au nom des Ahaggar pour solliciter la paix ») et celui réservé à « un chef qui rentre de dissidence parce que sa tribu s'est soumise sans lui et lui a forcé la main ». Mais il prend également soin d'ajouter:

«Un autre motif m’a aussi déterminé à supprimer toute parade c’est que la réception de Moussa en janvier 1904 a dépassé la juste mesure; l’impression qui en reste maintenant est que l'entrée de Moussa à In-Salah fut celle d'un triomphateur ou d'un puissant souverain: les indigènes du Tidikelt n'ont pas vu de soumission publique ni de cérémonie d'investiture; ils ont assisté à la lecture d'un traité de paix où la France et les Ahaggar prennent des engagements réciproques l...

En le prenant de haut avec Sidi ag Keraji, on fait, il est vrai, le jeu de l'amourpropre de Moussa, mais on établit aussi que les plus nobles des Touareg sont des sujets de la France, que l'on ne traite pas avec eux de puissance à puissance et

39. Cette lettre est reproduite dans l'appendice $n^{\circ} 1$ (Pièces annexées) du rapport de tournée (mai-octobre 1905) du capitaine Dinaux (AOM, 22H68). 
que, si des erreurs ont été commises, notre ligne de conduite tend à prendre une nouvelle orientation. 》

Finalement tout est ici énoncé. Ce qui est principalement reproché à Métois est d’avoir considéré et traité Mûsa et les Kel-Ahaggar non pas comme des sujets devant se soumettre à la France mais bien comme des partenaires placés quasiment sur un pied d'égalité. La manière dont Dinaux rend compte, dans le rapport de sa tournée 1905, des entrevues d'In-Salah en 1904 est à cet égard très significative :

"Moussa ag Amastane, chef du parti de la paix du Ahaggar. était venu à In-Salah en 1904 avec une suite nombreuse et imposante; il en était reparti après une réception brillante, et muni d'un traité en bonne et due forme. par lequel le chef d’annexe d'In-Salah et lui prenaient des engagements réciproques au sujet de la sécurité et de la répression des délits.

Moussa s’était aussitôt retiré dans l'Adrar, où il habitait depuis plusieurs années: connu et estimé dans tout le Sahara $[\ldots]$ il put augmenter encore sa popularité en annonçant partout $[\ldots \mid$ le pacte qu'il venait de conclure avec nous. et qu'il sut présenter comme un succès diplomatique, réservant l'indépendance des Touareg et leur garantissant le libre commerce."

D'où une situation pour le moins problématique. Selon Dinaux ${ }^{40}$, en effet : «Les Touareg voulaient bien reconnaître que Moussa avait "fait le bien et la paix" avec nous ; mais ils ne se considéraient pas comme nos sujets. Il en résultait une situation ambiguë, fausse, peu flatteuse pour nous malgré le contre-rezzou de 1902, et que les indigènes du Tidikelt jugeaient sévèrement. Car. conquis eux-mêmes très durement en 1900 et assujettis à l'impôt, ils déduisaient de notre attitude que nous ne pouvions ou n'osions agir de même vis-à-vis des Touareg. "

On comprend mieux dès lors que parmi les objectifs prioritaires assignés à la tournée de Dinaux figurent les points suivants: «rappeler à Moussa son rôle de chef soumis, [...] dire aux djemaâs ce que nous voulions et les conditions de leur soumission [...]». Soit " en un mot rendre la situation plus nette et le commandement plus facile ».

Cette détermination de Dinaux se retrouve en maints endroits de son important rapport de tournée. Avant même le début de celle-ci, dans un paragraphe intitulé "Préparatifs de départ". il note par exemple : «Au point de vue politique, je me bornai à écrire a Moussa en le traitant dès le début comme un chef soumis à la France et en le préparant à ma visite. " De même résume-t-il ainsi la philosophie politique de sa tournée:

40. Pour appuyer ce jugement. Dinaux se réfère a la tournéce effectuée en 1904 par le lieutenant Roussel. Dans son livre. Métosis laisse entendre. la aussi. sa différence quant a lappréciation politique de cette tournée: "Sans doute il n'y avait dans ces relations avec les Touareg qu'un tres vague sentiment de subordination. mais on peut voir cependant par la qu’il avait suffi dentamer la conversation avec eux pour ientendre, effet que des coups de fusils auraient difficilement produit... "(Mators 1905: 44). 
«Après l'apprivoisement des premières années, il convenait de parler en maître et de frapper l'esprit des Touareg... »

Et quand enfin Dinaux rencontre Mûsa, il croit déceler chez ce dernier, malgré une attitude conciliante, quelques réserves — au demeurant très compréhensibles ${ }^{41}$ — dans les propos du chef Kel-Ahaggar: «Toujours très correct, très déférent, très soumis dans la forme, il me donne peu à peu l'impression qu'il fondait le plus grand espoir sur sa visite au commandant militaire et qu'il comptait réussir à maintenir le statu quo de 1904. »

- Le problème de l'impôt. La question d'un impôt de soumission à appliquer aux Kel-Ahaggar est particulièrement révélatrice de l'opposition Métois/Laperrine. En 1904, à In-Salah, le capitaine Métois n'a pas exigé que les Kel-Ahaggar versent aux autorités françaises un quelconque impôt. Cette question n'est d'ailleurs même pas évoquée dans les dix points des «Instructions » alors remises à Mûsa agg Amastân. Certes, quand Laperrine transmet ce document aux autorités supérieures, il prend soin de justifier - mais en introduisant une réserve temporelle - la politique suivic par son subordonné. Dans la lettre qu'il rédige alors pour accompagner l'envoi à Alger des documents que lui a transmis Métois, on peut lire: "Avec des gens aussi orgueilleux et aussi jaloux de leur indépendance que les Touareg, il fallait éviter d'aller trop vite en besogne, aussi n'a-t-on pas été jusqu'à demander la soumission complète, on n'a pas parlé d'impôt qui est le signe palpable de la soumission. On a d'ailleurs fort bien fait car c'est également le signe de servage et les ennemis de la paix avec nous auraient profité de ce tremplin pour reprendre le dessus et rallier à eux toutes les tribus dites nobles » (AOM, 22H30).

Mais on peut se demander s'il ne s'agit pas là d'un passage dû pour l'essentiel aux rigueurs hiérarchiques. Métois se montre, lui, sans illusion sur l'opinion réelle de son supérieur: «... un malentendu regrettable m’avait amené à me montrer magnanime vis-à-vis de Moussá ag Amastan, alors que le commandant Laperrine aurait voulu lui faire payer l'impôt » (Métois 1995: 52). Significativement, un an plus tard, cette question de l'impôt, non pas en tant que ressource financière mais bien comme "signe palpable de la soumission ", est l'un des objectifs premiers de la tournée Dinaux. Lors de la rencontre entre cet officier et les notables Kel-Ahaggar à Tamanrasset, fut d'ailleurs confirmé le principe d'un impôt dû par les Kel-Ahaggar au gouvernement français. L'enjeu était de taille et bien qu'il ait donné son accord, par lettre envoyée à In-Salah, au principe d'un impôt, Mûsa était plusieurs fois intervenu auprès du capitaine Dinaux pour essayer de reporter à plus tard l'annonce de cette décision. Il craignait

41. Après avoir résumé les entretiens qu'il eut avec Mûsa en 1905, Dinaux écrit : «La situation que nous lui faisons, si brillante soit-elle, est bien terne à côté du grand royaume touareg indépendant qu'il avait rêvé, et dont sa première visite à In-Salah lui avait fait apercevoir le mirage» (souligné par nous). 
que celle-ci soit - en 1905 - mal acceptéc par les Kel-Ahaggar et exploitée par ses adversaires. In témoignent les nombreuses notations du capitaine Dinaux dans son rapport de tournée: " 27 juin 19(5) ] .. Moussa reparle de l'impôt offert par sa lettre au nom du Hoggar. demande qu'on n'en parle pas, le fruit n'étant pas encore mûr. » « 15 juillet 1905| ... Au sujet de l'impôt, non seulement Moussa s'y attendait. mais il l'avait offert par sa lettre d'In Zize. Il avait demandé seulement qu'avant d'en parler à ses gens on fasse remarquer que lui. Moussa. nétait pas sorti des conditions du traité d’In-Salah. Et c’est bien ainsi que jaa présenté la question... 》 (AOM, 22H68. souligné par nous).

Il sagissatit certes d'un impôt symbolique dont le montant ne fut d'ailleurs pas fixé alors. Mais les autorités coloniales y tenaient beaucoup en tant que signe manifeste de soumission. Le témoignage de Laperrine est sur ce point particulièrement explicite: "... il s"agissait de remettre au point la soumission de Moussa ag Amastane faite à In-Salah et de bien établir que c'était une soumission et non tune alliance. Il fallait faire accepter par les tribus un léger impôt qui fût une marque tangible de c'tle solumission" (Laperrine 1913: 405. souligné par nous).

- Atici ag Amellal. Dans le petit live que le capitaine Métois publie en 1906. Atici n'est pas particulièrement bien traité. La plupart des traits récurrents à la littérature de l'époque ${ }^{+2}$ s’y retrouvent (Métois 1995: 11 , 13 et 41). Et quand Métois narre la venue de Músa à In-Salah ê les entrevues qu'il eut alors avec ce dernier, aucune mention particulière n'est faite d'Atici. Pourtant il paraît évident qu’il fut alors question du sort à réserver à celui qui, à cette date, est encore l'amenâkal des Kel-Ahaggar. Dans certains écrits contemporains, il est d'ailleurs avancé que Métois aurait plus ou moins assuré à Mûsa qu'Atici ne serait pas inquiété par les autorités coloniales (cf. notamment Gautier 1906:11). C'est également ce qu avance Brunon (1967: 254) en se référant. sans indications précises, à certains propos de Métois. Mais c’est surtout dans nombre de documents

42. I.es "portraits" d'Atici seront encore plus chargés dans les nombreux écrits qui, par la suite, glorifieront lat conquéle coloniale au Sahara. Un des mérites de l'étude de Bostirot (1984) est d’atoir proposé sur cette période cruciale une analyse qui s inserit "a leneontre de lat version coloniale officielle" et d'avoir ainsi resitué la figure d'Atici hors de ces stéréotypes. el ce sans sombrer dans lanachronisme qui verrati en lui un héros anticolonialiste. Mais dans ce nécessaire et salutaire contrepoids deux points nous posent probleme. Si Atici fut en tout temps et en tout lieu un opposant irréductible. cest peut-étre aussi

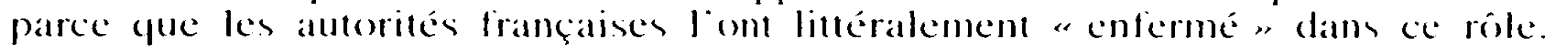
Enfin et surtout. il neest pas súr que lopposition MúsalAtici fut lonjours atussi complete que ne le suggere Brourgeot. Jusquen logs. deux laces en apparence contradictoires ont coexisté. I)"un coté. rivalité personmelle dlute pour le pouvoir) ef opposition politique fattitude ensers lat pénétratton françaises: de latutre. volonté (ou obligation ?). all monims pour Musa. de présenter une unité (monimale) des Kel-Ahaggar. de ne par etre combdere comme un diviseur. ("est hien la coexistence de ces deux aspects qui rend dificile lapprehension de cedte période. 
d'archives qu'est confirmé l'aman alors accordé à Atici. Métois lui-même nous a aussi laissé un écrit particulièrement révélateur. Dans cet article peu connu, paru en 1950, il confirme ce fait et surtout révèle que son étude de 1906 a été en maints endroits censuréc par ses supérieurs hiérarchiques:

« J'ai conté naguère les plus importantes de ces négociations continues. Cependant, je n'ai pas tout dit. À cette époque, j'avais encore des visées coloniales. Or, il y avait beaucoup de rivalités au Sahara, parmi les Français, rivalités de doctrines et rivalités de personnes. La prudence la plus élémentaire me commandait de taire un certain nombre de faits. D’autant plus que, pour publier, j'étais soumis au régime de l'autorisation ministérielle. Cependant, avant de me donner son accord, le bureau compétent soumit mon manuscrit à la Section d'Afrique, qui exerça une censure sévère, en marquant d'un trait de crayon bleu tous les passages à supprimer. Et il y en avait des passages! Les plus intéressants, bien entendu " (Métois 195()$: 12)$.

Et, de fait, cet article recèle quelques passages jusqu'alors inédits. Tel est notamment le cas du récit de la première entrevue entre Mûsa et Métois. Ainsi apprend-t-on que : «... Moussa prit le premier la parole pour me parler d'Etissi, dont il n'était, me dit-il, que le délégué " (ibid: 14). Atici amenûkal des Kel-Ahaggar et Mûsa simple porte-parolet.'? Il y a bien là de quoi remettre en cause la vision quelque peu rapide et réductrice qui domine la plupart des écrits consacrés à cette période. Quant à Métois, qui précise («...j’avais cru sage d'adopter la désignation de Moussa, faite par Cauvet, ... $)^{4+4}$, il répond ainsi à l'invite de Mûsa:

«Personnellement, je n’y verrais pas d'inconvénients. Je sais quel a été le rôle exact d'Etissi dans l’assassinat de Flatters et que, à ee moment-là, il a rempli tout son devoir. Je n'ai done aucune rancune contre lui. Tu peux lui dire de ma part qu'il peut vivre paisiblement dans l'Ahaggar et que, tant que je serai ici, personne ne fera quoi que ce soit pour lui créer des ennuis. Mais je ne suis pas encore sûr d'avoir convaincu tous mes chefs de la vérité. Et, si je proposais de reconnaître Etissi comme amenúkal, je craindrais d’être relevé de mon commandement. Dans ce cas, tout le travail que nous avons fait, toi et moi, en faveur de la paix, pourrait être annihilé. »

On est donc bien loin, ici, des stéréotypes coutumiers sur le sanguinaire et traître Atici. Surtout, à la lumière de tels propos, on peut mieux comprendre certains épisodes restés quelque peu énigmatiques. Tel est le cas de ce voyage dans l'Ajjer que Mûsa tient absolument à faire alors

43. Fst-ce à cela que songe Dinaux quand rapportant la nomination de Mûsa en tant qu'amenákal en 1905 il écrit : «... en consacrant à Moussa le titre d'amenokal, nous suscitons les jalousies de ceux qui, en 1904, l'avaient chargé seulement d'être l'ambassadeur de la paix. » Et parmi ceux qui ont (au moins) accepté la démarche de Mûsa ne doit-on pas faire figurer Atici et les siens?

44. Cauvet n'a jamais désigné Mûsa comme chef des Kel-Ahaggar. Il a simplement su (et Laperrine plus que lui encore) distinguer en Mûsa l'homme sur lequel la France pouvait s'appuyer. 
qu ${ }^{\circ}$ Hadj Bilou le presse de se rendre à In-Salah. On connaît l'explication qu'en donne Métois. Ce détour s’expliquerait par le fait qu'Atici, réactivant une vieille rivalité amoureuse, aurait fait dérober les habits de parade que Mûsa comptait revêtir pour son voyage à In-Salah. Version peu convaincante à vrai dire, et ce d'autant plus qu'on ne trouve aucune mention de cet imbroglio politico-sentimental dans le rapport de Hadj Bilou. Par contre, on y apprend que Mûsa et ses proches, en l'absence de Bilou. séjournèrent plusicurs jours dans le campement d'Atici. C'est accompagné de ce dernier que Mûsa rejoindra Bilou. Une grande assemblée se tient alors: y sont présents " les gens de l'Ahaggar, des Azdjer, ceux de Ghat, le caild Hassan et ag Keradji " (AOM, 22H30). Il sagit bien évidemment de savoir quelle attitude doit être adoptée concernant l'éventuel voyage de Mûsa à In-Salah. Or, Hadj Bilou prend soin de noter que seuls les Kel-Ajjer se montrent fermement opposés à cette initiative et quaprès son intervention «Etici. Amayas et ag Keraji sont rassurés et rentreront incessamment dans l'Ahaggar ". Peut-être y a-t-il là, de la part de Bilou. une vision quelque peu optimiste des événements. Ce qui paraît évident, en revanche, est bien que le détour de Mûsa s'explique avant tout par sa volonté de ne pas transformer sa démarche en une initiative purement personnelle. Il faut qu'elle soit approuvée ou du moins tolérée par le plus grand nombre possible de Kel-Ahaggar. Qu'Atici ait représenté, chez les Kel-Ahaggar, la ligne politique la plus fermement opposée à la pénétration française paraît évident. Mais, dans la situation connue par le Sahara central en 1904, pouvait-il réellement n'admettre aucun compromis et camper dans une position d'opposition irréductible ? Ce qui est sûr, c'est qu'après cette réunion dans l'Ajjer et alors que Mûsa se rend à In-Salah, Atici - comme l'annonçait Bilou - revient s"installer dans l'Ahaggar ${ }^{45}$.

De mai à septembre 1904, le lieutenant Roussel effectue une longue tournée dans la Téfedest et le nord de l'Ahaggar. Or. à cette occasion, Atici tente plusieurs fois d'entrer en contact avec lui. Par divers intermédiaires (notamment Seghir ag Bedda amghar des Tégehé Mellet ${ }^{+6}$ ) il fait comprendre à cet officier son désir d’établir un minimum de contact. Dans son important rapport de tournée (AOM, 22H67), Roussel le reconnaît plusieurs fois sans ambages et note (non sans un certain regret semblet-il) qu'obéissant à la ligne fixée sur ce sujet par Laperrine, il ne peut répondre que par la négative à de telles avances.

4.5. Ces allers et retours entre Ahaggar et Ajjer sont bien Évidemment tres significatifs. Selon les circonstances, selon ee qu il peut supposer de l'attitude des autorités d’In-Salah, Atici se rapproche ou s"éloigne de l'A haggar. Jusqu èn 1904 au moins, il ne peut ni ne veut śinstaller définitivement à l'écart de cette région car cela signifierait qu’il a abandomé son combat et que. de la rivalité qui loppose a Músa. ce dernier est désormais sorti vainqueur.

46. Amellal. le pere d'Atici et Anaba, appartenait aux Tegéhé Mellet alors que son épouse étatit. elle. Kel-(ihela. Sur l'utilisation que fit Atici de cette double filiation

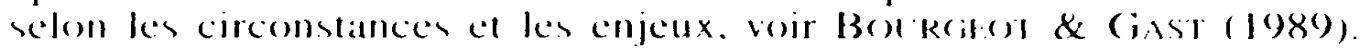


"II |Seghir ag Bedda|... me propose encore, comme il l'avait déjà fait plusieurs fois, de m’amener Ettici qu'il va voir: m'assurant que si je le désire Etticj viendra avec lui ; trop heureux de pouvoir se présenter. Je ne puis voir Ettici que nous tenons à ignorer et je lui réponds que je ne veux ni le faire demander, ni que lui Seghir [...] ne l'amène » (22 juillet 1904).

"Ettici, campé à quelques kilomètres seulement de Tazerouk, a lui aussi une autorité asse\% considérable [...]. Il tenait beaucoup, paraît-il, à se présenter, il était prêt à venir au premier signal, mais j'ai évité de parler de lui et il n'est pas venu " (3 août 19(1) .

Ainsi, tout semble indiquer qu'à ce moment là du moins, c'est bien davantage les officiers français qui, en appliquant strictement les décisions de Laperrine, refusent tout contact avec Atici et non l'inverse. Par là, Atici, qui fut effectivement le leader de la résistance à l'avancée française au Sahara central, se retrouve en quelque sorte enfermé dans son image d'opposant irréductible. Il est d'ailleurs très significatif de relever la différence de traitement qui est alors instaurée entre Atici et son frère cadet Anaba. Ce dernier a lui aussi été un farouche opposant et il a grandement participé au massacre de la mission Flatters. Pourtant, durant sa tournée de 1905, Dinaux le recevra plusicurs fois ${ }^{47}$. C'est Mûsa lui-même qui fut à l'initiative de ces rencontres. Dans son «Carnet de renseignements politiques ", Dinaux note ainsi la réponse qu'il donna alors: "Sur Anaba ; non seulement je le recevrai, mais je veux le voir. Atici est toujours mort pour nous" (AOM, 22H68).

Conscient que cette inégalité de traitement pose problème, Mûsa demandera d'ailleurs à l'officier français de ne pas évoquer l'attitude suivie envers Atici devant son frère Anaba «... qui s'effraierait, ne comprenant pas qu'on peut couper un doigt et laisser l'autre debout " (ibid.). En fait, Roussel et Dinaux ne font qu'appliquer à la lettre les directives de Laperrine. Ce dernier s'est toujours montré très clair sur l'attitude à adopter envers Atici. Dans un rapport du 30 octobre 1902, il affirmait déjà « Atici... sera toujours à la tête de nos ennemis irréductibles |... . Avec Atici et Si Abidin il n'y a rien à faire, ou plutôt une seule chose, leur envoyer un coup de fusil si jamais nous les avons à notre portée ». On est ici bien loin de l'attitude conciliante qu'adopta Métois à In-Salah et de l'aman qu'il accorda alors à Atici et ce, très vraisemblablement suite à une demande de Mûsa. Du coup les officiers qui, tels Roussel et Dinaux, suivent Laperrine se retrouvent fort embarrassés. Ils doivent dès lors se contenter d'ignorer Atici et de refuser - y compris quand il le demande - tout contact, toute entrevue avec lui. Sur ce point aussi, le différend opposant Métois à Laperrine ne date pas de 1904. Avant même la rencontre d'In-Salah, il s'est déjà manifesté lors d'un précédent retour d'Atici dans

47. Notamment le 4 juillet 1905 lors d'un palabre dont Dinaux écrit : «L'impression qui se dégage de ce palabre est bonne; Anaba a donné sa parole en public et son influence paraît grande ; Moussá est resté silencieux par déférence » (AOM, 22H68). 
l'Ahaggar. Dans une lettre à Cauvet datée du 15 juillet 1903. Laperrine notait : Les dissidents sont rentrés au Hoggar et Atici avec eux. Métois a l'air ravi, pour moi ça sonne mal. jattire son attention là-dessus, et le prie de ne plus recommencer le coup (hardenet en négociant avec cet assassin empoisonneur et traître " (AOM, 14X2).

- Chardenet ou Laperrine?. L'apparition de ce nom propre sous la plume de Laperrine est ici particulièrement significatif. Chardenet a commencé sa carrière militaire au Sahara. Dès 1894, licutenant au bureau des Affaires indigènes d'El Oued, il est chargé d'aller chercher dans la région de Ghadamès un groupe d’Ifoghas (parents de Si Othman) et de les installer dans la région du Souf. Après l'occupation du Tidikelt, c'est à lui que sera confiée la tâche de créer le premier poste des Affaires indigènes. Promu alors chef d'Annexe, il prendra part à toutes les opérations se déroulant alors dans cette région du Touat-Tidikelt. Appelé à l'état-major général (section Afrique). il quittera son poste d'In-Salah en février 1902. Mais si Chardenet apparaît ainsi dans la correspondance de Laperrine cela nest pas vraiment dû à ces seuls épisodes. somme toute classiques, de sa carrière saharienne. Chardenet est aussi le nom-symbole d'un conflit de style, et plus encore d'une profonde divergence politique. Pour Laperrine. Chardenet représente d'abord tous ces officiers qui, après un séjour plus ou moins bref "sur le terrain", ont rejoint les Bureaux d'Alger d'où ils impulsent, de manière bureaucratique, une politique sans rapport avec les réalités sahariennes. Dans une autre de ses lettres à Cauvet, datée du 14 avril 1902, Laperrine écrivait déjà : "Ton collègue Chardenet a l'air d'un homme très profond mais je ne comprends rien à sa politique ou plutôt je la comprends trop. jeter de la poudre aux yeux et arriver. Elle peut se résumer. Signer les travaux de ses officiers, se faire de la popularité en faisant taper sur les pauvres bougres inoffensifs et faire l'aimable avec les gens qui montrent les dents. Assaisonner le tout de rapports style pompeux mais pleins de blagues 》 (AOM, 14X2).

Mais les attaques de Laperrine ne se résument pas à cette dénonciation du carrièrisme propre. selon lui. à nombre de militaires ou de civils ayant à s'occuper du Saharate. Parmi d'autres, Chardenet est aussi à ses yeux le représentant type d'une ligne politique qu'il combat.

Ceci pourrait relever finalement de l’anecdote historique. Mais. élément capital pour nous. Métois fait plusieurs fois référence, et de manière fort élogieuse, à Chardenet et à la ligne politique suivie par ce dernier lors de son passage à In-Salah.

"Une période de trêve s'ouvrit quand le capitaine (hardenet prit le commandement de lannexe d'In-Salah. Lexcellent souvenir laissé par cel officier parmi les popu-

4x. ()n trouve dans la correspondance prive de laperrine de tres mombreusen el tres virulemes atlatgues contre les hureatlerates civils ou militatres qui. craignamt toute intatlive pronemt depuis Alger on Paris une politigue attentiste 
lations de la Tidikelt indique assez qu il avait compris les besoins du pays et que la politique agricole et commerciale qu'il avait inaugurée est celle qui convient le mieux à ces régions déshéritées. L’activité reprenait, la confiance renaissait, et les luttes stériles étaient momentanément laissées de côté. Le chiffre du mouvement commercial se haussait brusquement et les indigènes commençaient à croire au retour de l’âge d'or. Malheureusement. le capitaine Chardenet était bientôt absorbé par la colonne du Touat, puis quittait le territoire. Ses successeurs crurent devoir revenir à la politique pure " (Métois 1995: 13).

Ou encore ce passage dans lequel chacun des termes consacrés à Laperrine mérite décodage :

"Le commandant Laperrine m'ayant annoncé son retour à Aqabli pour les premiers jours de juillet. je me portai à sa rencontre pour lui rendre compte de l'orientation nettement pacifique que j’avais cru devoir adopter en son absence vis-à-vis des Touareg. Il voulut bien approuver les dispositions que $j$ avais prises. La satisfaction non déguisée que témoignait le chef de caravane. Akhmadou ag Chikat. que je pus lui présenter, indiquait assez qu elle produisait sur les Touareg un excellent effet et que ce retour à la politique Chardenet faisait la joie des indigènes" (ibid: 17).

Dans ces propos de Métois apparaît nettement qu'au-delà d'oppositions personnelles réclles il y a bien, à cette époque, divergence, au moins entre deux lignes politiques au Sahara ${ }^{49}$. C'est d'ailleurs ce qu'affirment certains des principaux acteurs de cette période. Quand Chardenet — dont Métois se réclame ouvertement - est en poste à In-Salah il a comme supérieur hiérarchique le général Cauchemez alors commandant des oasis sahariennes (Peyronnet 1930: 591). Or ce dernier est l'auteur, en 1902, d'un commentaire particulièrement révélateur. Donnant son avis sur un rapport de Iaperrine ${ }^{50}$, Caucheme\% écrit à propos de « la ligne de conduite que Laperrine propose de tenir vis-à-vis des Touaregs Hoggar »:

49. Dans une lettre adressée le $1^{" 1}$ avril 1902 au colonel Pein. Cauvet présentait ainsi le raid Cottenest : "... le résultat y est : attaque des Touareg et riposte subite. Nous sommes sur le pied de guerre; toutes les belles tartines de C... sont à vau-l'eau et le corps d armée aurait beau me prescrire de rappeler Cottenest, qu'il n'y a rien à faire" (in LeHI RAl:x 1935: 1(2). Nous n'avons pu consulter l'original de cette lettre mais il est très possible que ce C... désigne Chardenet.

50). À la date du 2 octobre 1902, Cauvet - alors chef d'Annexe à In-Salah - rédige une étude de 20 pages intitulée «Notice sur les fractions Touareg vivant dans l'hinterland de la Subdivision". Quand il transmet ce document aux autorités supérieures d'Alger. Laperrine y joint un important "Rapport sur la notice établie par le Chef d’annexe d'In-Salah " dans lequel il expose clairement la ligne de conduite qui, selon lui, doit être suivie vis-à-vis des nomades du Sahara central : "Le Touareg ne reconnaît que la force. Si vous hésitez à employer la force, si devant ses menaces ou ses pillages vous ne bougez pas, vous nusez pas de représailles, il vous considere comme un poltron et a pour vous le plus profond mépris. Si au contraire châtiant sévèrement les pillards, vous ne molestez pas les pacifiques et même vous vous montrez bons... alors ces gens sont fort étonnés. mais devant la preuve de votre force ils sont bien obligés d'admettre que vous vous montrez bons parce que vous le voulez bien et non par peur et vous 
"Une première méthode avait été essayée. c’est celle que j’appellerai la méthode douce : je l'avais préconisée lors de mon séjour dans les Oasis Sahariennes et je l'avais résumée ainsi aux officiers sous mes ordres: "Faciliter le courant commercial des Touaregs avec le Tidikelt. $|\ldots|$ en profiter pour entrer en relations avec ces nomades, les mettre peu à peu en confiance, ne pas se laisser rebuter par les obstacles et. le moment venu, proposer aux Touaregs de liquider le passé én leur laissant entendre que nous nous montrerions aussi accommodants que possible" $|\ldots|$.

Le commandant Laperrine reconnaît que ce système avait donné dés résultats, mais [...] il se montre surtout partisan de la force "Répondre à un rezzou par un contre-rezzou". mais comme ce dernier natteint jamais les vrais coupables, nous en arrivons à la guerre de représailles et dans la région qui nous occupe, celle-ci nous conduit à loccupation du Hoggar.

Fst-ce cela que veut notre Gouvernement ? | ... |

Avec le commandant Laperrine, nous sommes en plein rêve. en pleine fantaisie imaginative... »

L’intérêt d’un tel passage ne réside pas seulement dans l’exposé des deux lignes politiques qui s'opposaient alors au Sahara. Il est aussi un bon résumé de cette "méthode douce" selon Cauchemez, de " l'attitude timide » selon Laperrine que prônaient alors un certain nombre de civils et militaires notamment ceux qui avaient fait l'essentiel de leur carrière au sein des Bureaux arabes ${ }^{51}$.

Ces oppositions tactiques trouvaient d'autant plus à s'exprimer que le statut politico-juridique du Sud algérien n’était qu’imparfaitement défini durant cette période cruciale (1902-1905). Certes. par la loi du 24 décembre 1902 , les Territoires du Sud avaient été créés. Ce nouveau groupement territorial bénéficiait d'une administration et d'un budget distincts et se trouvait sous l'autorité directe du gouverneur général d’Algérie. Les pouvoirs de ce dernier étaient particulièrement étendus, y compris au plan militaire (voir Denis 1991: 97). Mais, il faudra plusieurs années pour réellement installer dans les faits un système de commandement fondé sur les principes exposés par la loi de 1902. Ainsi, ce n'est qu'en 1905. par les décrets du 14 août et du 12 décembre $5 ?$, que seront clairement

admirent." Cette ligne "dure" est aussi de fait une critique de toute politique attentiste de ce que laperrine dénomme. quelques pages plus loin. l'attitude timide. Attitude qui, en prônant "la défense demployer la force quoi qu“il arrive". est désastreuse, mais qui est cependant celle qui est préconisée - selon Laperrine -- par les autorités politiques et militaires en 1902.

51. Un exemple parfait en est fourni par le général (ate qui de 1901 a 1904, en lant que commandant du 19" Corps, se trouva à la tête de larmée d'Afrique (c.f. Pl:YRoNifle 19.30: 426). (Or, partisan d'une politique prudente et peu favorable a une extension territoriale all sud des oasis. (ave exprima plusieurs fois notamment a propos des raids de Collenest et Guillo-L ohan (AOM. 22H49) sat lotale désapprobation de la ligne politico-militare suivie par l aperrine. Aussi. son remplacement par le général Serviere plus proche des positions de laperrine. fut-il un signe parmi d'autres d'un changement politique d'importance.

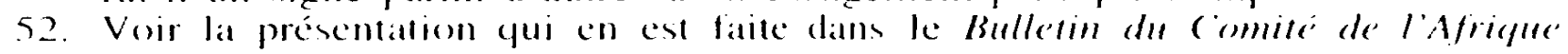
frangraise de janvier 1906) 
définis les rapports existant entre les commandants de territoires et le gouverneur général. C'est dans cette « sorte de vide juridique momentané » que le gouverneur Jonnart ${ }^{53}$ "s'efforce de prendre des initiatives qui établissent, d'avance, une pratique nouvelle [...] sans s'interdire d'y mettre sa marque personnelle, dont on dira qu'elle consiste à aller jusqu'aux limites extrêmes, acceptables par Paris, de l'expansion vers le Sud... » (Frémeaux 1993 : 98). Or, c'est également Jonnart qui a imposé la nomination de Laperrine et Lyautey à une armée d'Afrique qui «n'apprécie pas énormément qu'on fasse venir de France pour exercer d'importantes responsabilités, un chef d'escadrons du $7^{\mathrm{C}}$ Chasseurs et un colonel du $14^{\mathrm{e}}$ Hussards. Ni l'un ni l'autre n'ont jamais exercé de commandements importants en Afrique du Nord, ni ne sont passés par les Bureaux arabes, qui en sont considérés comme l'antichambre » (ibid.).

Mais ces deux hommes convenaient parfaitement aux options de Jonnart ${ }^{54}$. Comme le souligne J. Frémeaux, dont nous nous inspirons ici largement, les raisons de son choix apparaissent encore plus nettement quand on compare les "profils " de Laperrine et Lyautey à celui de leur prédécesseur qui n’était autre que le général Cauchemè. Ce dernier, homme des Bureaux arabes, était un proche de Révoil. Il n’était guère partisan de l'occupation du Sud qu'il jugeait, comme nombre d'autres officiers, trop coûteuse ${ }^{55}$. Début 1901 , conséquence directe d'un rapport établi par Cauchemez en septembre 190(), le ministère de la Guerre en arrivait à préconiser " l'évacuation rapide des oasis sahariennes les laissant à la garde des chefs indigènes jusqu'au jour où lè chemin de fer permettrait d'occuper le pays à moindres frais » (Hepp 1971: 142). Option qui sera finalement abandonnée grâce notamment à l'action résolue d'un des protecteurs de Laperrine: le général Serviére, commandant de la division d'Alger. Dès lors, il est tentant de voir, dans la disparition d'une équipe très liéce au Quai d'Orsay (Révoil, Cauchemez, Delcassé...) un recul d'influence de la «méthode douce» au profit d'une politique plus agressive, guidée par Étienne ${ }^{5 \hbar}$ et représentée sur le terrain par des hommes comme

53. Sur ce personnage et l'importance de son rôle politique en Algérie au début du siècle, voir AcifiRon (1968: 1003-1021 notamment).

54. Tout semble indiquer que c'est sur la proposition de Jonnart, appuyé par le général Servière, que I aperrine fut nommé (décret du 6 juillet 1901 ) commandant des Oasis sahariennes. Il en fut de même pour la désignation (10 septembre 1903) du colonel Lyautey pour commander le territoire d'Ain Sefra (Hr:P 1971 et FREMLALix 1993).

55. Opinion largement répandue à l'époque. Nombre d'auteurs trouvaient démesuré l'effort (notamment financier) consenti au Sahara au vu des bénéfices minimes que pourrait en retirer, sclon eux, la puissance coloniale française. Voir notamment DF: CHAMPFALX (1903).

56. Sur le rôle important qu'a joué Eugène Étienne, député, sous-secrétaire d'État aux Colonies et membre particulièrement influent du "parti colonial », voir AGFRON (1968 et 1978 : 150)-164 notamment). 
Jonnart. Lyautey et I aperrine. Or. tout indique que Métois penchait plutôt pour la "méthode douce »"

(On retrouve d'ailleurs fréquemment dans ces écrits lidée, exposéc dans le texte de Caucheme\%. que cést avant tout en favorisant le développement du commerce et en améliorant les conditions de vie des populations locales que lon pourra attirer les nomades du Sahara central. Option stratégique qui soinscrit dans la durée, qui ne revendique pas de hauts faits d'armes mais qui, selon ses partisans. permet d'établir des rapports de confiance réciproque. Chè Métois, cette option se combine avec une prise de parti. elle aussi explicite dans ses écrits. pour les couches les plus défavorisées des sociétés sahariennes ${ }^{5 x}$. S`y ajoutent également une vision extrêmement tranchée des relations entre monde arabe et monde touareg et. dans ce cadre, un jugement très critique sur liutilisation de religieux musulmans (du Touat-Tidikelt notamment) pour entrer en contact avec les Kel-Ahaggar. La question des rapports, obligatoirement conflictuels et hostiles sélon Métois, existant entre Arabes et Touaregs est évoquée dans tous ses écrits. Ainsi. dans un de ses articles. aprés avoir rapporté un récit tratiant de loorigine des relations établies entre habitants du Tidikelt et Kel-Ahaggar, il précise:

"Ce récit. d'autres me l'ont fait depuis, avec des variantes insignifiantes dans le détail. mais toujours avec cette idée mantresse invariable: la haine de l'Arabe. Ils lont sucée avec le lait. elle a fait l'objet de toutes les conversations l...l. I.es Touareg ont leurs préjugés. qui sont profondément enracinés. et leurs traditions. qui se transmettent de génération en génération. I a base des uns et des autres. cest. je le répète. la haine de l'Arabe. Un Arabe qui se risque chè cux n ést jamais sûr de revenir. Il le sait hien et leur rend largement haine pour haine" (Métois 1907: 3.39).

Chè Métois cette insistance sur l'état d’hostilité régnant entre Arabes et Touaregs s'accompagne toujours d’un éloge particulièrement appuyé

57. Sur un autre point encore Métois semble proche de Caucheme\% (e dernier. comme nombre d’autres militaires des Bureaux arabes. n'était pas un chaud partisan de l'occupation du Sahara central jugée périlleuse et onéreuse. Surtout. il refusait tout "coup de force " militate qui placerait le geuvernement devant le fait élabli. On retrouve une préoccupation semblable dans la lettre que Mélois rédige pour présenter l'accord d’In-Salah a ses supérieurs hiérarchiques (voir cidessus page 55). (Or. a celle date (février 19(1)4). Laperrine et les siens sont bien loin de ce légalisme républicain. Ils noont aucun doute sur lavenir proche et sont press par divers " coups de force " (comme le furent les raids cottenest et

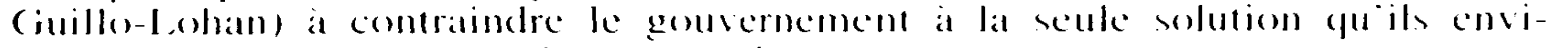
sagent : loceupation du Sahara central.

58. I.cur prise de position en taveut des populations serviles du Sahara central

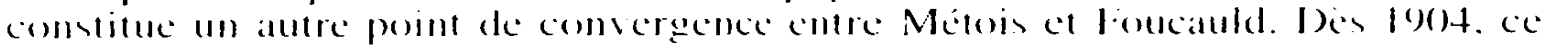
dernier se montreral particulierement élogieux ensers la pelitique mence sur ce sujet par le chef de l'Annexe d'In-Salats et lat citera comme un exemple a suir (1)(1) (.1111) 1903: 1.30$)$. 
des Ahl Azzi" Ces derniers - à la différence des populations qualifiées d'arabes - auraient depuis toujours entretenu des rapports particulièrement amicaux avec les Kel-Ahaggar dont le territoire ne leur a d'ailleurs jamais été interdit. Selon Métois, grâce à ses relations privilégiées, les Ahl 'Azzi ont assuré un rôle d'intermédiaires, de « ravitailleurs » entre le pays touareg et les oasis du Touat, et sont à l'origine de la fortune d'InSalah. Cette vision n'est pas sans incidence sur les choix qui peuvent s'opérer en ce début du siècle pour mieux pénétrer le pays touareg. Les militaires français tentent en effet de s'appuyer sur certains personnages du Touat-Tidikelt pour entrer en relation avec les Kel-Ahaggar et éventuellement les amener à eux. Ainsi Cauvet, durant son passage à In-Salah, a largement utilisé les services d'un cherif, Mouley Omar, caïd d'Akabli. Or, aux yeux de Métois, un tel personnage cumule deux inconvénients majeurs : celui d'être un religieux et un Arabe. D'où le choix qu'il fait de son concurrent direct: le caïd Bilou des Ahl 'Azzi. C'est dire qu'avec parfois des choix différents, les autorités coloniales ont parfaitement su utiliser les rivalités existantes tant entre les religieux du Touat-Tidikelt (Bourgeot 1984) qu'entre les différentes confréries (Pandolfi 1996).

\section{D'In-Salah à Tamanrasset (1904-1905)}

Pour Laperrine et les officiers qui suivent sa ligne politique, la rencontre d'In-Salah est loin de constituer une véritable soumission. Bien au contraire, nombre d'entre eux estiment que, dans le contexte de l'aprèsTit, Mûsa a pu et su présenter ces entrevues de 1904 comme une véritable victoire.

Il est très possible que ces officiers aient quelque peu noirci le tableau pour mieux imposer leur propre vision de la politique à suivre. Il n'en demeure pas moins que l'attitude de Mûsa dans les mois qui suivirent sa venue à In-Salah permet d'appuyer une telle opinion. Durant cette période, Mûsa s'emploiera à résider le plus loin possible d'In-Salah et à éviter tout contact avec les autorités françaises. C'est ainsi que, malgré les messages qui lui furent alors expédiés, il ne rencontrera pas Laperrine quand celui-ci réalisera une longue tournée en mars-juillet 1904. Certes, Métois (1906: 40) lui trouvera plusieurs "excuses" mais sans se faire d'illusion sur l'impression que laisse alors l’attitude de Mûsa : «... à la

59. Les Ahl 'Azri (ou Kel-(Ghezri dans l'Ahaggar) font partie de ces groupes de berbères sahariens qui formèrent les Almoravides, d'où leur nom générique de Mrabtines. Résidant au Touat-Tidikelt ils se prétendent originaires du Tafilalet. Ils entretiennent depuis longtemps des relations privilégiées avec les Kel-Ahaggar. À la demande de ces derniers, à la tin du XIX siècle. un certain nombre de Ahl 'Azzi sont venus s installer dans l'Ahaggar pour y cultiver, grâce au système des foggaras, les terrasses alluviales. Voir notamment Voinot (1909) et GAst (1986b). 
suite de sa tournée, le commandant I aperrine pouvait croire que la soumission de Moussa n'était qu"apparente "'s".

Ce que ne dit pas Métois éest que I aperrine l'accusera lui. le chef d'Annexe d'In-Salah, d'avoir tout fait pour que cette rencontre n'ait pas lieu. Dans des lettres adressées quelques mois plus tard à Cauvet, Laperrine écrit: "Crâce à Métois qui a manouvré en eau trouble, je n’ai pas vu Moussa qui s’est fait porter malade. D`ailleurs j’ai su que c’était déjà lui qui l'a empêché d’aller à Adrar comme il voulait le faire " (in lettre du 4 octobre 1904). "Furicux que j'aille chez. les Hoggars et les Ifoghas, il [Métois] a essayé de me jouer un tour en faisant que ma lettre à Moussa ag Amastane ne parvienne pas... " (in lettre du 4 octobre 1904).

Il est très possible que la rivalité existant entre ces deux officiers soit allée jusque-là. Reste cependant qu'un troisième homme intervient aussi ici : Mûsa agg Amastân. Si l’opposition Métois/Laperrine nous paraît un élément fondamental dans cette époque cruciale, c’est aussi parce qu'un personnage comme Mûsa, en fin politique, a su deviner cette contradiction et tenter d'en tirer profit. Quand, constatant cet état de fait, Dinaux écrit à Mûsa en avril 1905 , c'est sur le contenu même des entrevues d'In-Salah qu il revient avec insistance: "Comment! depuis plus d'un an que les autorités d'In-Salah vous ont ouvert les bras et vous ont reçu comme un fils de la France, vous n’avez cherché qu'à vous éloigner et à augmenter la distance entre nous. Que craignez-vous donc, et ne comprenez-vous pas votre intérêt et celui de vos gens ? [... | Mais comprenez bien qu'en faisant cela nous n’avons pas fait un traité entre deux puissances: nous l'avons fait parce que les Touareg sont maintenant les sujets du gouvernement français. »

Dès lors parce qu’In-Salah n’a pas constitué une véritable soumission, parce que Mûsa a su au mieux exploiter l'ambiguité de l'accord alors conclu, il est logique que Laperrine et les siens estiment capital dobtenir (ou réobtenir) la soumission des Kel-Ahaggar, et en le leur signifiant d'une manière explicite. Et ce d'autant plus que Laperrine et les siens craignent que les militaires français d'AOF n'attirent Mûsa vers eux. Dans les écrits de l'époque, revient comme un leitmotiv permanent le fait que Mûsa tiendrait plus qu’à tout aux Ifoghas. Or, il vient d’être alors décidé que ceux-ci seraient rattachés à l'AOF' d’où la crainte de voir Mûsa se diriger vers les autorités de Tombouctou. Cette inquiétude est d'ailleurs largement partagée par Métois et laperrine. I é premier rappelle ainsi qu én 1904. alors qu'Hadj Bilou se trouve auprès de Mûsa. "Hamadi. kebir des Kounta. convoquait Moussa dans l'Adrar pour lui parler d'une soumission au poste de Tombouctou. |... Hamadi faisait dire et disait lui-même que

6). Lt ce, alors que l'objectif de laperrine est aimsi énoncé dans son rapport de tournée: "Je jugeatis indispensable de visiler all plus lót les tribus mousellement soumises de façon a me rendre eomple sur plate de la sincérité ét de létendue de la soumission " (in I APfRRIN 1905: 37). 
si l'Ahaggar se soumettait aux Français d'In-Salah, il n'échapperait qu'aux répressions de ceux-ci, tandis que s'il faisait sa soumission à ceux de Tombouctou, il serait à l'abri de toute atteinte » (AOM, 22H30).

Quant au second, il exprime le 20 mars 1905, soit un an après InSalah, une crainte similaire dans une lettre à Cauvet: "Je suis assez embêté d'une fugue que ce dernier [Mûsa] a faite à Tombouctou où il a passé le mois de décembre 1904 comme me l'apprend une carte postale que me communique Cottenest. Je voudrais bien savoir ce qui a bien pu se discuter là-bas. Je crois qu'avec des promesses de pension on lui aura fait signer un papier de soumission à Tombouctou que l'on sortira à Paris pour prouver qu'il a fait une simple visite à In-Salah. D'ailleurs il a l'air de beaucoup plus tenir aux Ifoghas qu'aux Hoggars ou Azdjer. »

L'objectif principal de la tournée du capitaine Dinaux en 1905 est bien de confirmer sans aucune ambiguïté la soumission des Kel-Ahaggar et le ralliement - sans retour — de Mûsa. Il est d'ailleurs significatif qu'au début de sa tournée cet officier n'est pas certain de l'attitude qu'adoptera Mûsa. Si bien qu'il utilisera alors la rivalité Sidi agg Akaraji/ Mûsa pour attirer à nouveau ce dernier et reconfirmer son ralliement aux autorités françaises. Dans son rapport, Dinaux écrit : "Sidi s'est soumis dans l'unique espoir de supplanter Moussa, son rival de longue date, dont la politique et les jdées généreuses le blessent; nous ne l'avons nousmêmes investi que pour décider Moussa à se rapprocher de nous, et pour essayer de refréner les instincts de pillage des Taitoq » (Dinaux 19()7: 47).

Si les militaires français utilisent Sidi comme appât, le chef Taytoq trouve dans cette soumission un moyen pour lui de prendre sa revanche sur Mûsa. Dans ce jeu complexe où chacun des trois principaux protagonistes utilise les rivalités et contradictions existantes entre les deux autres, Dinaux retirera l'impression d'un "marché de dupes ». Dans le «Carnet de renseignements politiques » qu'il tient au jour le jour durant sa tournéc de 1905 , il se montre particulièrement clair sur ce point :

"... l'investiture de Sidi a été une faute, faute exclusivement due à la tactique de Moussa et au jugement que l'on portait sur lui. Si, il y a trois mois, j'avais connu Moussa comme je le connais aujourd'hui, il n'y aurait pas eu besoin de se servir de Sidi comme d'un appât pour décider Moussa à se rapprocher. Les Taytoq auraient été rendus à la confédération des Kel-Ahaggar, les Kel-Ahnet affranchis de toute refara en raison de la soumission première de Badjeloud et Sidi ag Keradji menacé d'internement à la première faute. Ç'eut été la vraie solution ; mais Moussa, en se dérobant et en jouant au sultan indépendant, l'a empêchée de se réaliser » (AOM, 22H68).

L'exploitation par Sidi agg Akaraji de la situation confuse qui règne alors obligera d'ailleurs le capitaine Dinaux à reinvestir Mûsa agg Amastân plus tôt que prévu, et ce «quoiqu'on ne soit pas au Hoggar »"1. Dès le

61. Cette double cérémonie de 1905, en ce qui concerne Mûsa, a son exact pendant dans la double nomination de Sidi agg Akaraji en tant qu'amghar des Taytoq. 
28 juin 1905 une première cérémonie d'investiture a lieu à In Ouzel. Au nom du gouvernement français. Dinaux confirme le titre d'amenákal de Mûsa. lui remet un burnous et lui propose un nouveau cachet. À In-Salah. Métois avait en effet remis à Mûsa un cachet portant l'inscription suivante : "amenûkal des imuhar " (littéralement "chef des Touaregs ") sans autre précision. En 1905, Dinaux annonce à Mûsa qu’il va lui faire envoyer deux nouveaux cachets portant (l'un en arabe, l'autre en tifinagh) le titre "amenûkal de l'Ahaggar ». Mûsa comprend très bien l’importance politique d'un tel changement comme l'explique Dinaux : "Mûsa dit que ce cachet lui a été donné à In-Salah : et. à son attitude, je suis forcé de lui expliquer que ce que je fais, ee n'est pas pour diminuer une puissance qu“il ne possède pas et que nous ne voulons pas lui donner; c'est pour répondre au désir du Colonel [Laperrinel qui n`avait pas autorisé le chef d'Annexe [Métois] à étendre son titre » (AOM, 22H68).

Par contre, il n'est pas alors fait mention d'un quelconque impôt. Dinaux se contente d'annoncer que c'est dans l' Ahaggar même, lors d'une cérémonie plus officielle, que seront abordées plus précisément les conditions de cette soumission des Kel-Ahaggar ${ }^{62}$. De fait. le 25 août 1905, en présence de Dinaux, se trouvent réunis à Tamanrasset la plupart des notables Kel-Ahaggar. Outre des nobles Kel-Ghela (Mokhammed ag Ghothman, Akhamuk ag Ihemma, Sughi, etc.) sont également présentes des délégations des principaux groupes tributaires: Dag-Ghâli, Aguh-ntahlé, Kel-Tazulet, Ayt Loayen, Ghelaydin... Là, Dinaux reconfirme la nomination de Mûsa. Il précise également que désormais les Kel-Ahaggar doivent verser la tiwse à Mûsa mais aussi payer un impôt au gouvernement français ${ }^{6.3}$. D'autres points sont également abordés : position par rapport à Atici. attitude des autorités françaises envers les Kel-Ajjer, nécessité de

Sidi a été une première fois investi le $1^{\prime \prime}$ mai 1905 à In-Salah. puis une seconde fois. selon les vocux mémes de Dinaux. le 24 mai dans l'Ahnet, en présence d'une quarantaine de Taytoq et Kel-Ahnet. La démarche suivie tant pour Músa que pour Sidi nous paraít sur un point très révélatrice de la ligne alors suivie: il faut que la soumission ait lieu au cour méme du territoire de ces chefs et en présence des représentants les plus éminents de leur eltebel respectif. Attitude que l'on pourra comparer à l'embarras de Métois quand il apprend que Laperrine a décidé d'entreprendre en $19(04$ une tournée dans l Ahaggar: "J'avais annoncé à Moussa quoon n’irait pas che\% lui " (Misols 1995: 36).

62. A ce propos, on relevera et thabile réponse de Músa et, dans la bouche de Sidi agg Akaraji, un bel exemple de celle parole voilée (voir ('Asistes 1987 el 1996) chere alux Touaregs: "A la remise du burnous. Moussa répond: "Je reste sur le pacte passé el je $n^{\circ}$ y changerai rien". Et Sidi riposte: "Le reg est uni pour tout le monde" faisant allusion a l"impot qu"il paye et dont on ne parle pas a Moussa" (D)NAlX 1907: A()M. 221168).

6.3. "Impót de principe" selon les termes mémes de Dinaux qui précise a la diemata réunie: "I 'impot n’est pas fixé : il sera léger parce qu"on comnail la situation du pays : des quon laura déterminé. Moussal le répartirat entre toutes les fratetions du pays" (AOM, 221368$)$. 
la «paix » pour assurer - notamment par la reprise du commerce - la prospérité de la région... Autant de sujets qui tendent tous à démontrer que désormais les Kel-Ahaggar et leur seul chef (Mûsa) sont soumis, et ce sans aucune ambiguité, à la France. Dinaux ne se contentera pas d'engagements verbaux. Non sans un certain volontarisme, en plusicurs occasions, il voudra démontrer aux Kel-Ahaggar que leur soumission a aussi des conséquences concrètes : opposition au désir exprimé par Mûsa de regagner l'Adagh, réquisition de chameaux auprès des Dag-Ghâli, etc. Le commentaire fourni sur ces décisions est d'ailleurs significatif :

"Moussa paraît contrarié de ces demandes et cherche à obtenir que l'on patiente et que l'on passe en touristes dans le Hoggar. Je lui fais comprendre que $|\ldots|$ si je n'avais pas besoin de chameaux, j'en demanderais quand même aux I)ag Rali. Il faut qu'ils confirment leur soumission par des actes $[\ldots]$.

J'ai eu l'impression plusicurs fois que Moussa me considère comme un gêneur [...]. C'est la première fois que l'on parle d'impôt, que l'on fait acte de commandement, que l'on séjourne dans le pays et Moussa ne peut croire, après les raids rapides du Colonel Laperrine et la situation obtenue par sa visite à In-Salah, que ma ligne de conduite actuelle soit approuvée par mes chefs " (AOM, 22H68).

Mais, quoi qu'il en pense, Músa devra céder devant toutes les demandes de Dinaux. Celui-ci pourra alors ainsi présenter les résultats obtenus à Tamanrasset: "En résumé, d’un côté, le titre d'amenokal consacré à Moussa, les refara dues à Moussa, l'impôt de principe payé au gouvernement. I) l'autre, la sécurité pour tous, le libre commerce sur nos marchés, le repeuplement et l'augmentation du bien-être dus à la paix. [...] Il se dégage de ce palabre une excellente impression qui, s'ajoutant aux résultats obtenus dans l'Adrar auprès d'A naba et des Kel-R'ela, permet d'affirmer que le Hoggar est en ce moment dans d'excellentes dispositions et a fait acte réel de soumission générale. "

Et de fait après cette nomination de $19(05$, bien plus qu'après les entrevues d'In-Salah (1904), les jeux sont faits dans l'Ahaggar. Durant dix ans, l'option politique représentée par Mûsa va aller en se renforçant. Mûsa lui-même verra son influence devenir de plus en plus déterminante. Certes, en 1916-1917, l'embrasement général du pays touareg touchera aussi l'Ahaggar prouvant, si besoin est, le caractère toujours contraint (et donc toujours plus ou moins temporaire) des soumissions coloniales. Il n'en reste pas moins que l'Ahaggar et Mûsa resteront malgré tout le pilier le plus solide, celui sur lequel prendront appui les militaires français dans cette période où vacille l'ordre colonial au Sahara. En ce sens, 1905 nous paraît bien une date capitale et la véritable conclusion (et conséquence) politique de la défaite de Tit. C'est bien à ce moment-là que se scelle pour une longue période le destin du Sahara central. Encore doit-on préciser que Mûsa et Laperrine sont loin d'avoir réalisé alors le rêve politique qu'à des niveaux différents tous deux poursuivaient : celui d'un 
grand territoire saharien placé sous leur autorité. L’ambition de Mûsa, celle pour laquelle il s'est montré prêt à devenir un "homme-lige » de la France, était d'être non seulement le chef de l'Ahaggar mais également celui de l'Adagh des Ifoghas, de l'Ajjer, voire de l'Air. Par bien des points, cette ambition était aussi celle de Laperrine.

Or, à cet égard, 1905 marque aussi un premier coup d'arrêt puisque, suite à la convention du 7 février 1905 (cf. Bouguetaia 1981), les Ifoghas se trouvent placés sous l'autorité de l'AOF échappant ainsi et à Mûsa et à Laperrine ${ }^{64 .}$.

Dans les écrits de l'époque, un thème récurent est sans cesse présent: les Ifoghas seraient les protégés de Mûsa et leur seul désir consisterait à intégrer son ettebel. Il y a là une présentation quelque peu rapide et partisane de la situation. Ainsi, au contraire de ce qui a souvent été affirmé, les Ifoghas ont présenté leur soumission aux autorités de l'AOF avant de se tourner vers celles d'In-Salah ${ }^{6.5}$. Mais, là aussi, l'analyse de cet épisode historique ne peut faire l'impasse sur les divergences politiques présentes au sein même du camp français. Comme l'a relevé Boilley (1993), les Ifoghas ont su profiter de cette situation pour tenter de construire un ettebel indépendant tant des Iwllemmeden que des KelAhaggar. Certes, comme l'indique Bourgeot (1995: 321), les autorités françaises n'avaient pas intérêt à voir Mûsa à la tête d'un grand ensemble touareg. Mais il est également vrai qu'en soustrayant les Ifoghas à l'Algérie, c'est aussi l'ambition jugée excessive de Laperrine qui est mise en échec. Les projets de ce dernier entraient alors en contradiction, y compris d'un point de vue strictement militaire (Frémeaux 1993), avec l'objectif principal qui était le Maroc plus qu'une extension vers le sud des territoires

64. Laperrine et les siens n'ont jamais véritablement admis cette "perte " des Ifoghas. Ils tenteront aussi de trouver une compensation vers l'Ajjer d'où, de fait, une connivence d’ambition avec Mûsa. Ainsi, dès 1905, prenant prétexte de ce que les Ifoghas étaient désormais rattachés à l'AOF et non à son ettebel, Músa avait demandé aux autorités coloniales françaises d’être nommé chef des Kel- $\Lambda$ jjer (AOM, 22H67). Proposition que Laperrine appuya dans une lettre du 25 septembre 1905, adressée au gouverneur général : "J'ai l'honneur de solliciter de votre haute bienveillance de rattacher les Azdjer à l'Annexe du Tidikelt et de décider que les tribus dont l’amenokal Moussa ag Amastane amènerait la soumission à la France seraient placées sous son commandement. C"est d'ailleurs ce que le commandant Cauvet lui avait promis implicitement et ce sera pour lui une compensation de la perte des Ifoghas de l'Adrar."

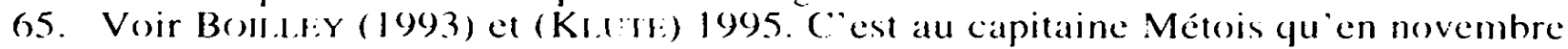
190.3 fut envoyée de la part des lfoghas une offre de soumission aux atutorités algériennes. Mais, a ce propos aussi. quelques divergences apparurent entre laperrine et le chef de l'Annexe d'In-Salah comme le révele une lettre adressée a cauvet par le premier nommé : le Soudan va étre furieux, je suis en train de reforger le rapport de Métois ou il y avait des considérations sur létendue démesurée que cette solumission donnatit at l'Annexe et qu'il tallatit prévoir pour l avenir en vue de l...l. ("était préparer le tremplin sur lequel le pere ("ase aturait dansé la gigue " din lettre du 2 décembre 1903). 
algériens. Les dernières lignes de l'important ouvrage publié en 1906 par Bernard et Lacroix sont, à cet égard, on ne peut plus claires : « À coup sûr, le Sahara ne vaut pas qu'on y dépense beaucoup d'hommes et beaucoup d'argent. [...] La question de la pénétration saharienne ne doit donc plus encombrer notre politique, et c'est vers les territoires autrement intéressants qui s'étendent à l'ouest de l'Algérie qu'il nous faut surtout porter nos regards ${ }^{66}$.

Opinion largement partagée par Lyautey, « ennemi de tout sacrifice au Sahara » (Frémeaux 1993: 103) et qui, sur ce point, n'hésitera pas à rappeler à l'ordre Laperrine, placé à partir du 12 décembre 1905 sous son autorité directe ${ }^{67}$. Ainsi, dans une note d'inspection relative à son subordonné (1906), Lyautey écrira : «... il serait utile, je crois, qu'il vînt une fois par an en France ou à Alger pour se rendre compte que le Sahara n'est pas une région indépendante et que la politique et les mouvements de la troupe doivent y être subordonnés à l'ensemble de la politique et des nécessités algériennes » (in Frémeaux 1993: 103) ${ }^{68}$.

Mais, malgré ces importantes restrictions, 1905 marque - au moins ponctuellement - la victoire de la ligne politique défendue par Laperrine qui prend désormais le pas sur celle qu'incarnait un officier comme Métois. Début 1905, suite à une décision de la hiérarchie militaire, Métois quitte In-Salah et rejoint à Alger le Service des Affaires indigènes. Laperrine n'est pas pour rien dans cette mutation comme le révèlent certains passages de la correspondance qu'il adresse à Cauvet:

"Il [Métois] m’écrit des lettres à peu près insolentes, et aujourd'hui je reçois une lettre de Moussa vieille de deux mois et de plus sans un mot d'explication,

66. Voir également l'article signé Henry de Castries dans le Bulletin du Comité de I'Afrique française d'août 1902. L'auteur y considère qu'on a largement surestimé l'importance politique du Sahara. Il souhaite que la pénétration saharienne soit stoppée et que les moyens financiers et humains qui y sont dépensés soient reportés sur le Maroc.

67. Voir $B C A F$ de janvier 1906. Que Laperrine soit placé sous les ordres de Lyautey est une décision significative. Elle le devient encore davantage si on relève que les hommes qui, par le même décret, sont nommés commandants militaires de Touggourt et du Sud tunisien sont deux militaires proches du commandant Rebillet. Or, pour ce dernier, "la diplomatie et le refus des "affaires" ont toujours eu le pas sur l'action militaire " (FRÉmEALX 1993: 103).

68. La tournée entreprise par Laperrine fin mars 1906 (Adrar-Taoudenni), sans aucune autorisation ni de ses supérieurs algériens ni des autorités soudanaises, exacerba encore davantage ses rapports avec Lyautey. Laperrine s'en confie d'ailleurs à Cauvet dans une de ses lettres: "Le général Lyautey trouve que je m'occupe trop des Touaregs et a demandé que l'on me fiche dehors; il n'a pas répondu à mes lettres particulières et a giberné pendant trois jours Gautier (Herr professor) à l'hotel de l'Oasis sur le thème suivant : il m'adore, m'aime beaucoup, me trouve parfait, mais ne veut plus entendre entendre parler de moi comme subordonné et déclare me lâcher à fond. Alors !!! Ces gens-là ont des ââmes (!) si complexes qu'il faut être Bourget lui-même pour s'y reconnaître » (in lettre du 10 juillet 1906: AOM, 14X2). 
simplement vu et transmis au bas du texte arabe. Jen ai plein le dos el je serai obligé de le débarquer ou de débarquer " 4 octobre 1904)"

"Je reçois des lettres charmantes de Métois mats il est trop tard je divorce. d'ailleurs la nomination de Dinatux à In-Salah aurait été signéc le 29 XI d'aprés un kélégrammè 115 janvier 1905$)$.

La carrière saharienne de Métois est terminéc. Toujours fidèle à sés convictions, il quittera l'armée avec le grade de lieutenant-colonel et fondera, en 1934, le parti républicain populiste qui publia le journal La rraie République (Humbert 1995). Il meurt en 1956 dans l'indifférénce générale. Silence significatif et qui commence dès 1905. Ainsi, dans son rapport de tournée (et ses annexes). Dinaux réussit le tour de force de ne pas citer une seule fois le nom de son prédécesseur. L_écriture hagiographique qui va régner par la suite ne fera que renforcer cet ostracisme ${ }^{70}$. Dans l'abondante littérature consacréc au Sahara central et à ses figures emblématiques, son nom et son rôle sont quasi systématiquement ignorés et même dans l'imposant (984 pages !) 《Livre d'or » publić par Peyronnet en 1930 il n'aura pas droit à la moindre notice.

La tournée Dinaux c’est aussi l’arrivée et l’installation de Foucauld à Tamanrasset (Pandolfi 1997b). Autant dire que les trois figures majeures (Laperrine. Foucauld. Músa) de ce qui va constituer la saga saharienne sont désormais en place. Du même coup, pour d'autres. tels Atici dans un camp et Métois dans l'autre ${ }^{71}$, c'est une plus ou moins longue période de silence et d’ignorance qui commence.

Centre d'anthropologie généralisée, Université de La Réunion.

69. I ans la méme lettre, Laperrine précise qu'il a informé L yautey de ses relations conflictuelles avec Métois: "L Lautey... a été furieux de l’attitude de Métois. Je crois que je serai obligé de le débarquer...".

70. A quelques tres rares exceptions pres, dont celle motable. de lehuraux (197(1). Ce silence occulte non seulement le roble de Métois at In-Salah mais également les autres facedtes de sa carriere saharienne: mission foureatu-I amy nombreux écrits consatcres at Sahara (ct. hibliographic). cte.

71. Il neest évidemment pas dans notre propos de comsidérer que le róle de ces deux personnages fut de la mente importance historigue. Nous coubons simplement relever ici que tous deux tigurent parmi les perdants de 1905. el que pour tous deux commence alore une période de relatif oubli. 
BIB LIOGR A PHIE

Archives d’Outre-Mer, Aix-en-Provence: Séries 22H30, 22H49, 22H67, $22 \mathrm{H} 68$ et $14 \times 2$ principalement.

AGERON, C. R.

1968 Les Algériens musulmans et la France (1871-1919), Paris, PUF, 2 tomes.

1978 France coloniale ou parti colonial?. Paris, PUF.

Al_AWJELY, G.

1975 Histoire des Kel-Denneg, Copenhague, Akademisk Forlag.

AOUAD-BADOLAL, R.

1993 «Le rôle de 'Abidine el Kounti dans la résistance nomade à la conquête française dans la Boucle du Niger (1874-1902)", Cahiers de l'IREMAM, $4: 35-48$.

BARRÉRE, G.

1990 «À propos de la tournée au Hoggar du L.t. Cottenest en 1902 », Le Saharien. $112: 7-12$.

BINHAZIRA, M.

1908 Six mois chez les Touaregs du Ahaggar, Alger, Jourdan.

BIRNARD, F.

1881 Quatre mois dans le Sahara. Journal d'une mission chez les Touareg suivi d'un apersu sur la deuxieme mission du Colonel Flatters, Paris, Delagrave.

BERNARI), A. \& LACROIX, N.

1906 La pénétration saharienne (1830-1906). Alger, Imprimerie algérienne.

BI:RNLS, E.

1970 «Récits historiques de l'Azawagh. Traditions des Iullemmeden Kel Dinnik", Bulletin de l'IFAN, XXXII, sér. B, n² 2 : 434-485.

BOHLLLY, P.

1993 «Aux origines de la question touarègue au Mali. Une exception coloniale: l'occupation pacifique de l'Adrar des Ifoghas », Islam et Sociétés au Sud du Sahara, $\mathrm{n}^{\circ} 7:$ 143-180.

Boliglietala, B.

1981 Les frontières méridionales de l'Algérie, Alger, SNED (Société nationale d'édition et de diffusion).

BOLiRGiFot, A.

1979 «Les échanges transsahariens, la Senusiya et les révoltes twareg de 1916 17 ", Cahiers d'Études africaines, XVIII (1-2), 69-70: 159-185.

1984 «Les mouvements de résistance et de collaboration en Ahaggar de 1880 à 1920 », Annuaire de l'Afrique du Nord. XXII : 479-499. 
199.5 Les sociétés muaregues. Nomadisme. identité, résistance's, Paris. Karthalat. BolRCilot, A. \& GAST, M.

1989 "Atici ag Amellal ". Encyclopédie berbere, VII : 1043-1046.

BRINON, R.

1967 "La découverte du Hoggar et le combat de Tit ", in Le Sahara: rapports et contacts humains ("Mémoires et Travaux de la Faculté d'Aix-enProvence 》 4.3): 22.3-2.56.

CASAJ'S, I).

1987 "Parole retenue et parole dangereuse chez les Touaregs du Niger». Journal de la Soriété des Africanistes, LVII (1-2): 97-108.

1996 "La parole voilée". Bulletin trimestriel des Amitiés Charles de Foucauld, $123: 21-24$.

Casthill. GatTlinara, G. C.

1992 I Tuareg attraverso la loro pessia orale, Roma. Consiglio Nazionale delle Ricerche.

CASTRIH:S, H. IP.

1902 "La politique du Sud en Algérie". BCAF (Bulletin du Comité de l'Afrique française), $8: 290-293$.

CAlvi:T, G.

1903 "Notice sur les fractions touareg du sud d'In-Salah", BCAF, XIII (1): $35-38$.

1945 Le raid du Lieutenant Cottenest au Hoggar, Marseille. R. \& J. Brunon.

CHAMPAXX. G. DI:

1903 A travers les oasis sahariennes, Paris. Chapelot.

CoTILNiST. Lt.

1988 "Tit 1902. Rapport de tournée ". Le Saharien, 105: 1-18.

DI:CRAH.N., Ph. \& ZICARIIII. F.

1994 Cirands Sahariens à la décomberte du "désent dés déserts". Paris. Donoël.

1)I.NS, P.

1991 L'armée française ate Sahara. Paris, L'Harmattan.

I)INAIX. ( a ap.

1907 "Rapport de tournée (0.3-0.5 all 29-10) 1905), B(AF, Re'nserignements (o)lenicut. I (pp. 11-19). 2 (pp. 42-51) et 3 (pp. 62-72).

I) (RO) . J. M.

1993 I. exploranion du Salara. Arles. Actes Sud.

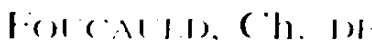

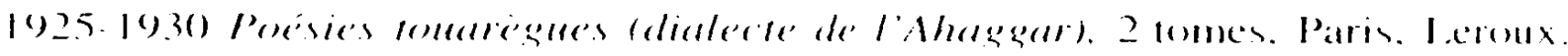


1984 Textes touaregs en prose (dialecte de l'Ahaggar), Aix-en-Provence, Edisud (1"éd. 1922).

1993 Carnet de Beni Abbès, Paris, Nouvelle Cité.

1994-1995 "Chez les Touaregs (Taitoq, Iforas, Hoggar) ", Bulletin trimestriel des Amitiés Charles de Foucauld, 115:9-18;116:6-13;117:15-24; $118: 8-15$.

Folreal, F.

1902 D'Alger au Congo par le Tchad, Paris, Masson.

Frémeaux, J.

1993-1995 L'Afrique à l'ombre des épées, 1830-1930. Des établissements côtiers aux confins sahariens, 2 tomes, Paris, Service historique de l'Armée de terre.

GAST, $M$.

1986a "Histoire des Kel-Ahaggar », Encyclopédie herbère, III : 282-303.

1986b "Ahl 'Azzi », Encyclopédie berbère, III : 320-322.

1993 "Un document inédit à propos du massacre de la mission Flatters en 1881 », Cahiers de I'IREMAM, $4: 49-56$.

GALTILR, E. F.

1906 «Du Touat au Niger», La Géographie, XIII: 5-18.

GRI:VOY., D.

1994 Lés méharistes français à la conquête du Sahara, Paris, I.'Harmattan.

Hr:P, M.-A.

1971 "Le général Laperrine, organisateur de la pacification du Sahara », Revlte" historique des Armées, 2 : 142-167.

HL:MBI:RT, J. C.

1995 "Préface", in F.-A. Mítols, La soumission des Touareg du Nord. Calvisson, Gandini.

KI.UT:, G.

1995 «Hostilités et alliances. Archéologie de la dissidence des Touaregs au Mali ", Cahiers d'Études africaines, XXXV (1), 137: 55-71.

LAPERRINE, H.

1905 «Une tournéc dans le Sud de l'annexe de Tidikelt », BCAF/Renseignemènts coloniaux, 2 : 37-46.

1913 «La pacification des Touaregs: Notes et souvenirs », Questions diplomatiques et coloniales, 35 (pp. 393-407; 517-529; 651-659), et 36 (pp.74$81 ; 137-146)$.

LEHLRALX, L.

19.35 Le conquérant des oasis. Colonel Th. Pein. Paris, Plon.

1970 "Les pacificateurs du pays touareg", Le Saharien, $54: 20-32$. 
MARII:I., A.

196.5 Le's confins saharo-tripolitains de la Tunisie. Paris, PUIt. 2 vols.

MARTY, P.

1920 Énde's sur l'Islam el le's tribus du Soudan. Paris. I.eroux. 3 vols.

Métols, I'.-A.

1903 "Le Tidikelt. Sa valeur économique", Bulletin de la Société de Ciéographie d'Alger, VIII : 618-624.

1907 "Ain-Salah et ses dépendances". Annales de (jéographie. XVI : 337-349.

1950 "L investiture de Moussa ag Amastan amenokhal des Touareg de l'Ahaggar", Vert et Rouge, 29: 10-15.

1995 La sommission des Touareg du Nord. 1900-1904, Calvisson, Candini (1" éd. 1906).

NICOIAS. F:

1944 "Folklore twareg. Poésies et chansons de l'A rawarh", BIFAN (Bulle'tin de I'Institut français d'Afrique noire). VI (1-4): 1-46.3.

1950 Tamesna. Paris, Imprimerie nationale.

NoRRIS, H.T.

1975 The Tuaregs. Their Islamic Legacy and its Diffusion in the Sahel. Warminster, Aris \& Phillips.

PANDOLFI, P.

1996 "Tijaniyya et Touaregs du Sahara central à la fin du XIX“ siècle : la délégation de 1892 ", Islam et Soriétés au Sud du Sahara, 10: 25-41.

1997a Touaregs de l'Ahaggar. Parenté et résidence chez les Dag-Ghali, Paris, Khartala (à paraître).

1997 b "Sauront-ils séparer entre les soldats et lés prêtrés ?". Sur l'installation du Père de Foucauld dans l'Ahaggar ". Journal des Africanistes, 67 (2): 49-71.

PEYRONNIT, R.

1930 Livre d'or des officiers des affaires indigènes (18.30-1930), tome II : Notices et biographies, Alger. Imprimerie algérienne.

POTIH:R. R.

1951 La Mission Foureate-Lamy, Paris, Íditions Union française.

RI:IHA:I.1. I:

1931 Carmet de route de la Mission saharienne Fonereate-Lamy. Paris. Plon.

RICHIR, A.

1924 l.es Oulliminden, Paris, Larose.

Scmontl, général

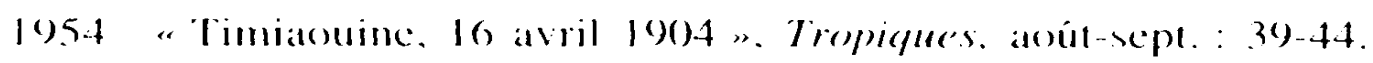


SIRRMNIN, M. D):

1994 «Portraits". Bulle'tin de's Amitiés Charles de Foucauld, 116: 19-22.

VAl.HT, R.-V.

1927 Le Sahara algérien. Étude de l'organisation administrative, financière et judiciaire des Territoires du Sud. Alger, La Typo-Litho.

VOINOT, L.

1909 Le Tidikelt, Oran, L. Fouque.

\section{RÉSIJME}

Dans less premières années du $x^{\prime \prime}$ siècle, suite à la défaite militaire connue par les Touaregs Kel-Ahaggar au combat de Tit (1902), la France s'installe au Sahara central. Dès lors un des principaux obstacles à la liaison Maghreb/Soudan est levé. Longtemps l'histoire consacrée à cette période cruciale s'est cantonnée dans un discours hagiographique et répcititif. L'examen de documents d'archives jusqu'ici peu exploités permet de mieux cerner les stratégies mises en couvre et les lignes politiques divergentes qui se sont alors manifestees - au moins au niveau tactique -. tant chey les Kel-Ahaggar que che\% les militaires francais. La reconstruction de ce pan d'histoire met au jour non seulement les alliances et le's divisions propres aux deux camps en présence mais également l'empirisme qui a souvent dominé la politique de: pénétration coloniale dans cette région.

\section{ABSIRAC I}

In-Salah, 1904/Tamanrassest, 1905: Twe acts of submission by the Kel-Ahaggar Tuareg. - Early in the 20 th century, following the battle of Tit in 1902, when the? French defeated the Kel-Ahaggar Tuareg, France extended its authority into the central Sahara. This did away with one of the major obstacles to contacts between North Africa and the Sudan. For a longtime, the history of this key period has been locked up inside a repetitious, hagiographic discourse. Archives, which have not been much studied to date, shed light on the strategies and divergent political lines pursued, at least tactically, by the Kel-Ahaggar and French military officers. Reconstructing this history brings to light the alliances and divisions on each side; and it draws attention to the empiricism that often prevailed in colonial policy about how to penetrate this region.

Mots clés/Keywords: Ahaggar, Laperrine, Métois, Músa agg Amastán, politique coloniale, Touaregs/colonialism, Ahaggar, Laperrine, Métois, Mûsa agg Amastân, Tuareg. 